Wilfrid Laurier University

Scholars Commons @ Laurier

Biology Faculty Publications

Biology

$10-2020$

\title{
Mechanistic insights into strigolactone biosynthesis, signaling and regulation during plant growth and development
}

\author{
Kaiser Iqbal Wani \\ Aligarh Muslim University \\ Andleeb Zehra \\ Aligarh Muslim University \\ Sadaf Choudhary \\ Aligarh Muslim University \\ M Naeem \\ Aligarh Muslim University \\ M. Masroor A. Khan \\ Aligarh Muslim University
}

See next page for additional authors

Follow this and additional works at: https://scholars.wlu.ca/biol_faculty

Part of the Agricultural Science Commons, Agriculture Commons, Biology Commons, Cell Biology Commons, Molecular Genetics Commons, and the Plant Biology Commons

\section{Recommended Citation}

Wani, K.I., Zehra, A., Choudhary, S. et al. Mechanistic Insights into Strigolactone Biosynthesis, Signaling, and Regulation During Plant Growth and Development. J Plant Growth Regul (2020). https://doi.org/ 10.1007/s00344-020-10234-w

This Article is brought to you for free and open access by the Biology at Scholars Commons @ Laurier. It has been accepted for inclusion in Biology Faculty Publications by an authorized administrator of Scholars Commons @ Laurier. For more information, please contact scholarscommons@wlu.ca. 


\section{Authors}

Kaiser Iqbal Wani, Andleeb Zehra, Sadaf Choudhary, M Naeem, M. Masroor A. Khan, Christian Danve Castroverde, and Tariq Aftab 
1 Mechanistic insights into strigolactone biosynthesis, signaling and regulation during plant

2 growth and development

3 Kaiser Iqbal Wani ${ }^{1}$, Andleeb Zehra ${ }^{1}$, Sadaf Choudhary ${ }^{1}$, M. Naeem ${ }^{1}$, M. Masroor A.

4 Khan $^{1}$, Christian Danve M. Castroverde ${ }^{2}$ and Tariq Aftab ${ }^{1 *}$

$5 \quad{ }^{1}$ Department of Botany, Aligarh Muslim University, Aligarh - 202 002, India

$6 \quad{ }^{2}$ Department of Biology, Wilfrid Laurier University, Waterloo, Ontario, Canada

$7 \quad$ Corresponding author email: tarik.alig@gmail.com 


\section{Abstract}

19 Strigolactones (SLs) constitute a group of carotenoid-derived phytohormones with butenolide

20 moieties. These hormones are involved in various functions, including regulation of secondary

21 growth, shoot branching and hypocotyl elongation, and stimulation of seed germination. SLs also

22 control hyphal branching of arbuscular mycorrhizal (AM) fungi, and mediate responses to both

23 abiotic and biotic cues. Most of these functions stem from the interplay of SLs with other

24 hormones, enabling plants to appropriately respond to changing environmental conditions. This

25 dynamic interplay provides opportunities for phytohormones to modulate and augment one

26 another. In this article, we review our current mechanistic understanding of SL biosynthesis,

27 receptors and signaling. We also highlight recent advances regarding the interaction of SLs with

28 other hormones during developmental processes and stress conditions.

29 Keywords: Carotenoid-derived phytohormone; butenolide moieties; Phytohormone crosstalk;

30 Strigolactone biosynthesis; Strigolactone receptors; Strigolactone signaling 
Strigolactones (SLs) comprise a novel class of phytohormones first discovered as

33 germination inducers of various parasitic plant species (Cook et al. 1966; Kohlen et al. 2011).

34 Their name originates from their role in stimulating Striga (parasitic witchweeds) germination,

35 and from their characteristic lactone ring structure. The first isolated Striga seed germination

36 inducers were strigyl acetate and strigol from Gossypium hirsutum L. (Cook et al. 1966).

37 Retrospectively, SLs were first indicated as phytohormones through their presence as unknown

38 graft-transmissible signals that suppressed Pisum sativum shoot branching (Beveridge et al. 1994).

39 Signal-deficient mutants showed a hyper branching phenotype that was independent of known

40 phytohormones, like cytokinins and auxins (Koltai 2014).

Two research groups then independently identified SLs as new phytohormones regulating

42 the shoot branching phenotypes (Gomez-Roldan et al. 2008; Umehara et al. 2008). Plant shoot

43 branching is inhibited by endogenous SL production or exogenous SL application in these hyper

44 branching mutants (Umehara et al. 2008) (Fig. 1). Root and shoot extracts of various species,

45 including Arabidopsis, contain various types, combinations and levels of SL molecules

46 (Goldwasser et al. 2008; Koltai and Beveridge 2013; Kapulnik and Koltai. 2014; Saeed et al. 2017;

47 Bürger and Chory 2020). To regulate shoot branching, root-derived SLs are mainly transported to

48 shoots through the xylem (Kohlen et al. 2011; Borghi et al. 2016). Since the discovery of SLs as

49 phytohormones, extensive research has revealed novel insights about their diversity, biosynthesis

50 and signaling. Because of their important roles in plant growth and development, SLs can

51 potentially be used for crop improvement. For example, mutating the SL biosynthetic gene

$52 H T D 1 / D 17$ increases rice yields, which contributed to the "Green Revolution" since the 1960s

53 (Wang et al. 2020a). 
SLs are characterized by their butenolide moieties - lactones with a 4-C heterocyclic ring

55 structure (Omoarelojie et al. 2019). These hormones are at the forefront of plant science research

56 because of their diverse biological roles, ranging from growth and development to interactions

57 with other organisms (Agusti et al. 2011; Cook et al. 1966; Toh et al. 2012; Domagalska and

58 Leyser 2011). The synthetic SL analog GR24 is an important tool in investigating the functions of

59 SLs in plant physiology (Arite et al. 2009). It has been most useful in species without known SL

60 biosynthetic/signaling mutants and its application reverses SL biosynthetic but not signaling

61 mutant phenotypes (Gomez-Roldan et al. 2008; Umehara et al. 2008).

62 Although initially considered to be detrimental to plants since they enhanced parasitic plant

63 germination (Cook et al. 1966), SLs were later considered beneficial since they also mediate

64 arbuscular mycorrhizal (AM) fungal colonization (Akiyama et al. 2005; Besserer et al. 2006).

65 Moreover, they initiate AM fungal hyphal branching even before host root infection (Akiyama et

66 al. 2005). SLs also interact with rhizobia and affect nodule formation in leguminous plants,

67 reflecting their diverse roles in biotic interactions (Foo et al. 2014). Apart from their functions in

68 regulating plant symbiotic relationships, SLs may mediate defences against pathogens (Torres-

69 Vera et al. 2014).

In addition, SLs can effectively alleviate various abiotic stresses (Fig. 1), such as salt and

71 drought stresses (Ma et al. 2017; Van Ha et al. 2014; Lu et al. 2019). In Arabidopsis thaliana, SLs

72 can regulate adaptive responses, such as stress-induced changes in stomatal density and closure

73 (Van Ha et al. 2014). In their study, SL-deficient plants were hypersensitive to such stresses (Van

74 Ha et al. 2014). Exogenous SL application rescued drought-sensitive mutant phenotypes, while it

75 augmented the drought tolerance of wild type (WT) plants (Van Ha et al. 2014). 
Other hormones interact with SLs to regulate various physiological processes, enabling plants to respond to changing environmental factors, such as nutrient availability, shading and temperature (Cheng et al. 2013). For example, auxins work together with SLs to control shoot branching patterns (Hayward et al. 2009, Bennett et al. 2016, Ligerot et al. 2017). SLs and abscisic acid (ABA) work together during abiotic stresses (Ren et al. 2018). Moreover, ethylene and SLs act antagonistically to control hypocotyl growth (Yu et al. 2013).

\section{Strigolactone biosynthesis: From humble pigment beginnings}

85 SLs and SL-like compounds have a conserved lactone structure consisting of three rings (ABC86 rings) connected through an enol ether bridge with a fourth methyl butenolide or furanone moiety

87 (D-ring) (Al-Babili and Bouwmeester 2015; Yoneyama et al. 2018). The region connecting the core $(\mathrm{ABC})$ with the D-ring acts as the bioactiphore (Zwanenburg et al. 2009). Endogenous SLs

89 are classified into two main types (strigol and orobanchol type) based on whether the $\mathrm{C}$ ring is $\alpha-$

90 or $\beta$-oriented (Cui. 2014). Strigol and orobanchol are canonical SLs as both have A, B, C, and D-

91 rings (Butler. 1995); around 23 types of canonical SLs have been characterized in root exudates

92 (Xie et al. 2010). Certain SL-like compounds are considered non-canonical, because they lack the

93 A, B and/or C-ring; however, they still possess the D-ring bonded to the rest of the molecule (Alder

94 et al. 2012; Boyer et al. 2014; Waters et al. 2017). Non-canonical SLs include certain synthetic

95 and natural compounds like methyl carlactonoate (MeCLA), avenaol and Yoshimulactone Green

96 (Abe et al. 2014; Kim et al. 2014; Tsuchiya et al. 2015). The structural diversity in canonical SLs

97 stems from various $\mathrm{AB}$ ring system modifications, including epoxidation, hydroxylation, 
98 ketolation and oxidation (Bhattacharya et al. 2009). This wide structural diversity involves many

99 SL biosynthetic genes (Saeed et al. 2017), homologs of which have been found in algae and

100 bryophytes (Delaux et al. 2012).

Several studies have elucidated the molecular mechanism of SL biosynthesis. The 102 involvement of the carotenoid pathway was reported using fluridone, an inhibitor of carotenoid 103 biosynthesis (Matusova et al. 2005). SL biosynthesis has also been investigated using certain 104 carotenoid catabolic mutants (Matusova et al. 2005), and different branching mutants such as $P$. 105 sativum ramosus (rms) mutants (Johnson et al. 2006; Beveridge et al. 1994), Arabidopsis max 106 (more axillary growth) mutants (Sorefan et al. 2003) and Petunia decreased apical dominance 107 (dad1, dad2, dad3) mutants (Snowden et al. 2005). Gene cloning, reciprocal grafting experiments 108 and mutant analysis implied that SLs are synthesized from carotenoids and are transported 109 acropetally (Ongaro et al. 2008). SL biosynthesis initially occurs in the chloroplasts (Alder et al. 2012; Saeed et al. 2017) 111 involving DWARF27 (D27/ $\beta$-carotene isomerase), which requires iron as a cofactor (Lin et al. 112 2009). D27 catalyses $\beta$-carotene isomerization by acting on its $9^{\text {th }}$ chemical bond, changing its 113 configuration from trans- $\beta$-carotene into 9-cis- $\beta$-carotene (C-40) (Alder et al. 2012). These 114 carotenoids have a 40-carbon skeleton with an extended conjugated double bond system (Moise 115 et al. 2014). Downstream of D27, carotenoid cleavage dioxygenases (CCDs) convert carotenoids 116 into apocarotenoids (Auldridge et al. 2006; Waters et al., 2012a; Hou et al. 2016), which are then 117 modified by other CCD enzymes (Alder et al. 2008). Oxidation of various carotenoid precursors, 118 resulting in specific double bond breakage, yields various compounds like ABA, SLs and retinal 119 (a conjugated chromophore) (Felemban et al. 2019). The Arabidopsis genome encodes about nine 120 different CCDs (CCD1-9), five of which are 9-cis-epoxycarotenoid cleavage dioxygenase 
121 (NCEDs) involved in ABA biosynthesis (Tan et al. 2003). In addition, various enzymes encoded 122 by MAX genes (MAX1, MAX3 and MAX4) regulate SL biosynthesis in Arabidopsis (Ruyter-Spira 123 et al. 2013). ABA itself may also regulate SL biosynthesis, because ABA-deficient maize (vp14) 124 and tomato (notabilis) mutants showed lower seed germination (Matusova et al. 2005). In molecular detail, CCD-catalysed SL biosynthesis produces intermediates that are further 126 oxidized by cytochrome P450s (Matusova et al. 2005). Two known CCDs (CCD7 and CCD8) act 127 progressively in the pathway; CCD7 is encoded by MAX3 and its orthologs RMS5 and D17/HTD1 128 (Booker et al. 2004), whereas CCD8 is encoded by MAX4 and its orthologs RMS1, D10 and DAD1 129 (Arite et al. 2007). 9-cis- $\beta$-carotene is converted by CCD7 into 9-cis- $\beta$-apo-10-carotenal (C-27) 130 and $\beta$ ionone (C-13) (Waters et al. 2012a). 9-cis- $\beta$-apo-10-carotenal is then converted by CCD8 131 into Carlactone (CL), a possible mobile intermediate containing two rings (A and D) along with 132 the enol ether bridge and an SL-like carbon skeleton (Alder et al. 2012; Seto et al. 2014). CL is 133 produced by intra-molecular rearrangement of 9-cis- $\beta$-apo-10-carotenal, which suggests that each $134 \quad \beta$-carotene molecule produces a single SL molecule (Alder et al. 2012; Seto et al. 2014). CL has 135 similar properties as SLs, such as stimulating seed germination of Striga hermonthica, and is a 136 putative intermediate during the biosynthesis of other SLs (Alder et al. 2012). Seto and colleagues 137 (2014) used ${ }^{13} \mathrm{C}$-labeled CL to detect its conversion into SLs in vivo. Conversion of exogenous CL 138 into SL has been reported in rice, suggesting that CL is the precursor of endogenous SLs (Seto et 139 al. 2014). Remarkably, Baz et al. (2018) reported that a new product 3-OH-carlactone is formed 140 in vitro from 9-cis-3-OH- $\beta$-apo-10'-carotenal by the action of D27, CCD7 and CCD8. They also 141 showed 3-OH-carlactone formation in planta by expressing rice and Arabidopsis CL biosynthetic 142 genes in Nicotiana benthamiana leaves (Baz et al. 2018). 
144 Bouwmeester 2015). CL (with a complete D ring) acts as the common precursor of all SLs;

145 however, it needs further modifications since it lacks the B and C rings (Alder et al. 2012). CL is

146 then converted into carlactonoic acid (CLA) by the cytochrome P450 monooxygenase enzyme

147 MAX1 in Arabidopsis (Abe et al. 2014; Zhang et al. 2014). Booker et al. (2005) demonstrated the

148 role of MAX1 (CYP711A1) in CLA synthesis, by reciprocal grafting experiments in A. thaliana.

149 In these experiments, the excessive branching phenotype of max4 (ccd8) mutant scions were

150 eventually reversed by grafting with wild type MAX1 root stocks (Booker et al. 2005). The

151 conversion of CL into CLA in vitro using recombinant MAX1 protein inside yeast microsomes

152 further clarified the function of MAX1 (Abe et al. 2014). MAX1 catalyses back-to-back oxidation

153 of CL at C-19, first forming 19-hydroxy-CL and then CLA (Abe et al. 2014). CLA has been

154 reported to accumulate in Arabidopsis roots, including those in atd14 and max2 mutants (Abe et

155 al. 2014). Endogenous CLA has also been reported in rice plants, and exogenous CLA is converted

156 into SLs using the $d 10-2$ rice mutant (Abe et al. 2014). When provided with ${ }^{13}$ C-labelled CLA,

157 d10-2 mutant root exudates subsequently accumulated ${ }^{13} \mathrm{C}$-labelled 5-deoxystrigol and orobanchol

158 (Abe et al. 2014). In Arabidopsis, CLA is similarly converted into 5-deoxystrigol and 4-

159 deoxyorobanchol (4DO) (Abe et al. 2014). 5-deoxystrigol is the simplest SL as it lacks hydroxyl,

160 acetyloxyl and other oxygen-containing substituents (Awad et al. 2006; Yoneyama et al. 2008). It

161 is found in both monocots (Awad et al. 2006) and dicots (Yoneyama et al. 2008), indicating it as

162 the precursor of all SLs. 5-deoxystrigol then undergoes either allylic hydroxylation (to strigol or 163 orobanchol) or homoallylic hydroxylation (to sorgomol) (Rani et al. 2008: Xie et al. 2010). Further

164 modification of sorgomol - oxidation of its hydroxymethyl group followed by decarboxylation -

165 results in the formation of sorgolactone (Xie et al. 2010). CLA can also undergo methylation 
166 (through an unknown methyl transferase enzyme) and be converted into the methyl ester MeCLA

167 (SL-LIKE1) (Seto et al. 2014). Interestingly, the conversion of CLA into MeCLA is MAX1-

168 independent as confirmed by Arabidopsis mutant analyses (Abe et al. 2014). Another enzyme LBO

169 (Lateral Branching Oxidoreductase) acts downstream of MAX1 to convert MeCLA into the

170 recently identified hydroxymethyl carlactonoate involved in shoot branching (Brewer et al. 2016;

171 Yoneyama et al. 2020)

172 Recently, a carotenoid-derived molecule zaxinone has been shown to negatively regulate

173 SL (4-deoxyorobanchol) biosynthesis in rice under phosphate (Pi) limiting conditions (Wang et al.

174 2019). This was confirmed by increased SL content in zaxinone synthase (zas) mutant seedlings

175 under Pi stress and enhanced Striga germination stimulation potential of zas root exudates (Wang

176 et al. 2019). This was similarly observed in tomato root exudates under Pi-deficient conditions

177 (Lopez-Raez et al. 2008). Enhanced seed germination vigour coincided with increased SL levels,

178 which then decreased upon phosphate restoration (Lopez-Raez et al. 2008).

\section{Strigolactone signaling cascade: A tale of binding, derepression and hydrolysis}

181 Phytohormone perception relies on a well-defined receptor system. Just like jasmonate, auxin and 182 gibberellin signaling (Schwechheimer and Willige. 2009: Dharmasiri et al. 2005; Katsir et al.

183 2008), SL signaling involves polyubiquitination and proteasomal degradation. The SL signaling 184 cascade involves three important components: (1) an $\alpha / \beta$ fold hydrolase called D14 in rice (Arite 185 et al. 2009), (2) an F-box leucine-rich protein called MAX2/D3 (Stirnberg et al. 2002; Johnson et 186 al. 2006) and (3) a repressor protein called D53 belonging to the SMAX1-like (SMXL) protein 187 family (Jiang et al. 2013; Stanga et al. 2013). The SL receptor protein D14 is activated after ligand 
188 binding, leading to its interaction with other molecules to form a signaling complex; hormonal 189 signal transduction is followed by subsequent hydrolysis of the bound SL, deactivating the 190 hormone (Marzec et al. 2016).

Various SL-insensitive mutants were analysed to identify different SL signaling 192 components (Seto et al. 2014). AtD14/D14/DAD2 are the orthologous SL receptors in A. thaliana, 193 Oryza sativa and Petunia, respectively (Waters et al. 2012b; Arite et al. 2009; Hamiaux et al. 194 2012); gene mutations result in a SL-specific phenotype that is not reversed by GR24 treatment 195 (Arite et al. 2009). These gene orthologs encode proteins similar to the soluble gibberellic acid 196 (GA) receptor GID1 (GIBBERELLIN-INSENSITIVE DWARF1) (Ueguchi-Tanaka et al. 2005). 197 These receptor proteins have a conserved catalytic triad consisting of Ser, His, and Asp (Zhao et 198 al. 2013). GR24 undergoes hydrolysis, most probably due to catalytic triad activity (Kagiyama et 199 al. 2013). The Petunia receptor DAD2 loses its catalytic activity with a Ser-to-Ala substitution 200 (DAD2:S96A) in the triad (Hamiaux et al. 2012), leading to loss of receptor interaction with the 201 F-box protein, thereby suppressing shoot branching (Hamiaux et al. 2012; Marzec et al. 2016). 202 GR24 undergoes very slow hydrolysis with DAD2, but the dad2 mutant phenotype is not reversed 203 by the resulting products (Zhao et al. 2013). This confirms DAD2 involvement in SL signaling, 204 with the hydrolytic process being more important than the end products (Seto and Yamaguchi 205 2014).

In rice, the SL hormone-D14 receptor interaction results in SL cleavage and subsequent 207 production of a "covalently linked intermediate molecule" (CLIM) bound to D14 (Bythell-Douglas 208 et al. 2017). Unlike other phytohormones, SL signaling depends upon hormone degradation. In 209 detail, binding of D14 with SL leads to nucleophilic attack, resulting in SL ligand dissociation into 210 two molecules: (1) the $\mathrm{ABC}$ ring portion called $\mathrm{ABC}$-formyltricycliclactone (ABC-FTL) and (2) 
211 the remaining part with the D-ring called hydroxymethylbutenolide (HMB) (Nakamura et al.

212 2013). ABC-FTL is released while HMB remains covalently attached to the D14 receptor; this

213 HMB-D14 intermediate is called CLIM (Yao et al. 2016). This reaction changes the D14

214 conformation, allowing it to interact with downstream signaling components (Marzec et al. 2019).

215 SL signaling proceeds from the interaction between the receptor D14 and F-box leucine-

216 rich protein MAX2/D3/RMS4 (orthologs in A. thaliana, Oryza sativa and Petunia, respectively)

217 (Hamiaux et al. 2012). MAX2 forms a part of the Skp-Cullin-F-box containing (SCF) E3 ubiquitin

218 ligase complex (Hamiaux et al. 2012; Zheng et al. 2014; Zhao et al. 2014). Mutations in these

219 orthologs lead to SL insensitivity, confirming their crucial role in SL signaling (Marzec et al. 220 2016).

This SCF complex targets the D53 and D53-like SMXL repressor proteins for proteasomal 222 degradation (Jiang et al. 2013; Zhou et al. 2013; Bennett et al. 2016). In Arabidopsis, SMXL6-8 223 have been proposed to be $D 53$ orthologs, as they regulate shoot branching and other SL-controlled 224 processes (Soundappan et al. 2015; Bennett et al. 2016; Ligerot et al. 2017). Due to its EAR motifs, 225 D53 is expected to interact with TOPLESS-related (TPR) transcriptional corepressor proteins 226 (Smith and Li. 2014). This D53-TPR complex may then repress SL target gene expression (Smith 227 and Li. 2014). The D53 repressor also interacts with the D14 receptor; upon GR24 treatment, D53 228 undergoes SCF complex-directed degradation (Smith and Li. 2014). The ligand-induced 229 conformational change in D14 allows the receptor to recruit SMXL7 into the SCF complex (Liang 230 et al. 2016). SMXL7 functions both transcriptionally and non-transcriptionally, but the molecular 231 events after its degradation have not been clearly elucidated (Waters et al. 2017; Bythell-Douglas 232 et al. 2017). In O. sativa, the major regulator of plant architecture Ideal Plant Architecture 1 (IPA1) 233 acts downstream of the D53 repressor, regulating SL-induced gene expression (Song et al. 2017). 
234 IPA1 is repressed by D53 in vitro and in vivo, which represses its transcriptional activation function 235 (Song et al. 2017).

Several engrossing hypotheses have been proposed to explain the evolution of ligand and

237 signaling specificity by D14 and D14-like receptor proteins. In parasitic plants, D14-like proteins

238 - closely related to D14 proteins - act as receptors of host-exuded SLs, representing a case of

239 convergent evolution (Tsuchiya et al. 2015; Conn and Nelson. 2015). These subfamilies of D14-

240 like proteins also include sub functionalized proteins that respond to other ligands, such as

241 karrikins and other D-lactone-containing compounds (Waters et al. 2012b; Saeed et al. 2017).

242 Perception of both SLs and karrikins also require the MAX2 F-box protein (Zhao et al. 2015).

243 However, it is unknown how MAX2 discriminates between the two pathways to generate different

244 responses, because F-box proteins tend to be indiscriminate when recruiting target proteins

245 (Nelson et al. 2011; Nakamura et al. 2013). Wang et al. (2020b) proposed that in Arabidopsis, both

246 SL and karrikin signaling pathways converge at SMXL2, as it acts as their common target for

247 polyubiquitination and degradation in a D14- or KAI2-dependent manner.

Different lines of evidence support the model that SL signal transduction occurs as a result

249 of SL binding/hydrolysis-induced conformational changes in the D14 receptor. For example,

250 thermal destabilization of the D14 receptor is initiated by GR24, which depends on an intact D14

251 catalytic triad (Waters et al. 2015). GR24 also promotes the physical interaction between

252 MAX2/D3 and D14, with MAX2/D3 further destabilizing the D14 receptor (Waters et al. 2017;

253 Zhao et al. 2014). Interestingly, D14-D3 association in $O$. sativa is a bit more responsive to $2^{\prime} \mathrm{R}$

254 stereoisomers of SL analogs compared to 2'S stereoisomers (Zhao et al. 2015). Furthermore, there

255 are no major structural differences between D14 and apo-D14, when associated with 5-hydroxy-

256 3-methylbutenolide, 2, 4, 4, trihydroxy-3-methyl-3-butenal or SL (Nakamura et al. 2013). 
Recently, several modes of SL-D14 interaction have been determined, but it is unclear

258 how D14 functions with D3 in ubiquitinating the D53 repressor. D3 has a C-terminal $\alpha$-helix that

259 exists in either engaged or dislodged forms (Shabek et al. 2018). The engaged form enables D14

260 and D3 binding with a hydrolysed SL intermediate, while the dislodged form recognizes

261 unmodified D14 and prevents its enzymatic activity (Shabek et al. 2018). The D3 $\alpha$-helix helps

262 D14 in recruiting D53 in a SL-dependent manner, which then activates the hydrolase (Shabek et

263 al. 2018). The self-induced D14 degradation by SLs (through MAX2) limits their own signaling

264 through a negative feedback loop (Chevalier et al. 2014; Koltai 2014).

265 Controversially, this CLIM model has been challenged by various experimental evidence.

266 CLIM cannot be accommodated in the D14 active site due to its very small electron density;

267 instead, iodine (I) in the crystallization reagents is suspected to bind the active site (Carlsson et al.

268 2018). D14-mediated SL hydrolysis is also too sluggish after SL treatment, in sharp contrast to the

269 rapid degradation of target proteins (D53/SMXLs) (Seto et al. 2019). Therefore, the rapid response

270 of SLs cannot be entirely explained by this CLIM model. Instead, it has been recently reported that

271 binding of a complete SL molecule, not a hydrolysed one, initiates the active D14 receptor

272 signaling; D14 then hydrolyses SL molecules only after completing the pathway (Seto et al. 2019).

273 Kinetic analysis of the AtD14-catalysed hydrolysis of 5-deoxystrigol detected two hydrolytic

274 products, $A B C-F T L$ and HMB, as described earlier (Hamiaux et al. 2012). The $K_{\text {cat }}, K_{m}$ and $V_{\max }$

275 values were found to be $0.12 \mathrm{~min}^{-1}, 4.9 \mu \mathrm{M}$ and $4.0 \mathrm{nmol} / \mathrm{min} / \mathrm{mg}$ protein, respectively (Seto et al.,

276 2019). In addition, 3,6'-dihydroGR24, which has a single bond instead of a double bond in the enol

277 ether bridge, is not hydrolysed by the SL receptors in rice and Arabidopsis (Umehara et al. 2015).

278 Furthermore, D14 catalytic activity is quite low for debranones (SL analogs without the enol-ether 
bridge), but these analogs interestingly yield the same results as GR24 (Scaffidi et al. 2014). These observations raise questions about the role of hydrolysis (by D14) in SL signaling. be proposed (Yao et al. 2016). In molecular detail, the D14 conformational change enlarges the catalytic pocket, allowing SL movement into this pocket and then closing the helical lid domain

284 (Shabek et al. 2018). When a SL molecule binds to the D14 receptor protein, D14 initially attains 285 an unstable conformation due to interruption in the catalytic triad formation (Yao et al. 2016). In 286 this changed conformation, the D14 receptor interacts with other components to carry out the SL 287 signaling cascade (Fig. 2). After activation, D14 (through the surface of its rearranged lid domain) interacts with the F-box protein MAX2/D3 and then D53/SMXL repressor binding occurs around 289 the region of the Asp loop (Seto et al. 2019). After D53/SMXL degradation, the D14 catalytic triad 290 is again reconstructed, which performs the important hydrolysis step, resulting in SL deactivation 291 (Seto et al. 2019). This hormonal degradation mechanism is also found in other hormonal pathways 292 (like GA) and is very important for hormone homeostasis (Yamaguchi. 2008). that the initial role of SLs was AM fungal recruitment to facilitate more efficient nutrient uptake; 297 this symbiotic association was present in land plants about 360-450 million years ago (Waldie et 298 al. 2014; Simon et al. 1993). Remarkably, SLs are found in algae and SL application results in 299 rhizoid elongation - a response also reported in liverworts and mosses belonging to bryophytes 300 (Delaux et al. 2012); however, it is most probably independent of MAX2 (Waldie et al. 2014). In 
301 higher plants, MAX2-independent SL signaling has also been reported. Minute GR24 302 concentrations can inhibit root growth in the $\max 2$ mutant (Shinohara et al. 2013). In charophytes, 303 a D14 member is more closely related to the KARRIKIN INSENSITIVE2 (KAI2) receptor than 304 to canonical D14 proteins (Waldie et al. 2014; Waters et al. 2012b). It might be possible that SLs 305 use this receptor instead of MAX2 to initiate their response (Waldie et al. 2014). The D14 and 306 MAX2 gene clades arose quickly when land plants emerged, with $D 14$ probably appearing due to 307 duplication in the clade, while another duplication within D14 resulted in the evolution of the D14308 LIKE2 group (Waters et al. 2012b; Waldie et al. 2014). These duplication events correlate with 309 varying functions as land plants diversified. D53 protein evolution also follows a similar pattern.

310 The $D 53$-like genes in mosses have higher similarity to SMAX1 than to D53/SMAXL7 clade; these 311 clades were then subjected to further duplications (Zhou et al. 2013). Intriguingly, the entry of

312 MAX2 into the SL pathway has not been fully elucidated. It is postulated that MAX2 was initially 313 involved in AM colonization only and its role in SL signaling evolved later (Challis et al. 2013);

314 this is supported by the $d 3$ rice mutant which cannot be colonized by AM fungi (Waldie et al. 315 2014).

\section{Strigolactone receptors: Highly conserved in diverse plant species}

318 The SL receptors have a conserved $\alpha / \beta$ hydrolase functional domain (Bennett and Leyser 2014), 319 which was first identified in the SL-insensitive O. sativa d14 mutant (Arite et al. 2009). Orthologs 320 were eventually identified in Petunia (Hamiaux et al. 2012), pea (de Saint Germain et al. 2016) 321 and Arabidopsis (Waters et al. 2012b). According to Arite et al. (2009), D14 homologs are found 322 in diverse plant clades, such as Marchantia polymorpha (bryophytes), Selaginella 
moellendorfi (pteridophytes) and gymnosperms. These homologs belong to the D14-like

324 subfamily, whereas angiosperm genes are grouped into the D14 subfamily of the $\alpha / \beta$-hydrolase

325 superfamily (Arite et al. 2009). Proteins of these subfamilies similarly possess a conserved

326 catalytic triad, a nucleophilic residue and an acidic residue, but have quite different sequences

327 (Nardini and Dijkstra. 1999; Arite et al. 2009). The $\alpha / \beta$ hydrolase superfamily also includes the

328 acetylcholinesterase (AChE) enzyme (responsible for acetylcholine metabolism) and the inactive

329 gibberellic acid receptor (Holmquist et al. 2000).

D14 (without any prefix corresponds to the $O$. sativa receptor) acts as a receptor as well as

331 an enzyme, differentiating it from other plant hormone receptors (Hamiaux et al. 2012). It has a

$332 \alpha / \beta$ hydrolase functional domain containing the Ser-His-Asp catalytic triad, forming its ligand

333 binding pocket, and $4 \alpha$ helices forming its cap (Kagiyama et al. 2013). It consists of 318 amino

334 acids, and a homolog called D14-like is also reported in the rice genome (Arite et al. 2009. The

335 rate of SL hydrolysis in vitro is as low as $\sim 0.3$ molecules per minute, suggesting that bioactive SL-

336 derived signal production is not its primary function (Snowden and Janssen. 2016). Consistent

337 with this, neither the intermediate molecule 2,4,4-trihydroxy-3-methyl-3-butenal nor the end

338 products of SL hydrolysis (tricyclic lactone and HMB) act as signals for shoot branching

339 suppression (Waters et al. 2017).

The SL receptor in A. thaliana (AtD14) is evolutionarily conserved (Waters et al. 2012b;

341 Arite et al. 2009); just like the rice D14 receptor, it consists of a catalytic triad and possesses both

342 receptor and enzyme functions (Hamiaux et al. 2012). The structure of the AtD14-D3-ASK1

343 complex showed a portion of the hormone covalently bonded with the receptor through two amino

344 acids in the triad (Yao et al. 2016). When the receptor conformation changes, an $\alpha$ helix domain

345 increases in length, while another $\alpha$ helix domain unfolds and forms a loop (Yao et al. 2016). Four 
$346 \alpha$ helix domains form the lid of the receptor, which probably functions in destabilizing the SL

347 receptor upon hormone attachment (Zhao et al. 2015; Snowden and Janssen 2016). The enzymatic

348 active site also decreases in volume resulting in closure (Fig. 3). Therefore, this indicates that D-

349 ring separation is difficult without complex dissociation, and could explain the sluggish enzyme

350 activity (Snowden and Janssen 2016). In Arabidopsis, the AtD14L/KAI2 protein is 51\% identical

351 and $75.9 \%$ similar to AtD14, but is instead involved in karrikin signaling; unsurprisingly, AtD14L

352 and AtD14 belong to different phylogenetic clades (Waters et al. 2012b).

356 solved its structure by X-ray crystallography and its lid consists of $4 \alpha$ helices, connected by a $\beta$

357 hairpin to the core. A strongly hydrophobic cavity between the lid and the core can easily

358 accommodate known SLs (Hamiaux et al. 2012). The authors further reported that when GR24 is

359 present, DAD2 interacts with the F-box protein PhMAX2A (the Petunia MAX2 ortholog). GR24

360 then undergoes hydrolysis upon DAD2 interaction, but mutations in the catalytic triad leads to loss

361 of enzymatic activity and failure to interact with PhMAX2A (Hamiaux et al. 2012). The prolific

362 branching phenotype of $d a d 2$ mutants has also been observed in dadl (CCD8) and dad3 (CCD7)

363 biosynthetic mutants (Napoli et al. 1996). DAD2 locally controls shoot branching, as confirmed

364 by grafting and genetic studies (Simons et al. 2007; Hamiaux et al. 2012). The branching

365 phenotype of biosynthetic mutants is reversed by grafting with wild type root stocks; however, this

366 reversion does not occur in $d a d 2$ mutants, suggesting that $D A D 2$ is not involved in SL biosynthesis

367 (Simons et al. 2007). 
The SL receptor in Hordeum vulgare (barley) is encoded by the $H v D 14$ gene, which consists of a 1055-bp coding sequence with two exons (Marzec et al. 2016). The approximately

370303 -amino acid HvD14 protein also contains the conserved $\alpha / \beta$-hydrolase domain between amino 371 acids 57 and 295 (Kagiyama et al. 2013). Unsurprisingly, it has great structural similarity, high 372 sequence conservation, and comparable secondary domains to the rice D14 ortholog ((Marzec et 373 al. 2016). In $h v d 14 . d$ mutants, the Gly at position 193 is substituted by Glu (Marzec et al. 2015); 374 this residue is present in the $\alpha \mathrm{D} 2 \alpha$-helical domain, which constitutes the cap surrounding the 375 active site along with $\alpha \mathrm{D} 1, \alpha \mathrm{D} 3$ and $\alpha \mathrm{D} 4$ (Kagiyama et al. 2013).

Zheng et al. (2016) reported that the woody perennial plant Populus trichocarpa has two 377 highly identical (91.7\%) and similar (95.9\%) homologs PtD14a and PtD14b. They showed that $378 P t D 14 a$ is $79 \%$ identical and $89.1 \%$ similar to $A t D 14$, while $P t D 14 b$ is $77.5 \%$ identical and $89.1 \%$ 379 similar to AtD14 (Zheng et al. 2016). The crucial Ser-His-Asp catalytic triad is conserved in both 380 PtD14 homologs at positions 96, 246 and 217 (Zheng et al. 2016). In terms of gene expression, 381 PtD14a transcript levels are higher compared to PtD14b, with very low co-expression between 382 them (Zheng et al. 2016).

The probable SL receptors in parasitic weeds were more difficult to identify, because the 384 phenotypes could not be dissected genetically (Toh et al. 2015; Tsuchiya et al. 2015). 385 Subsequently, a group of $\alpha / \beta$-hydrolases ShKAI2s/ShHTLs (S. hermonthica KARRIKIN 386 INSENSITIVE2/ HYPO-SENSITIVE TO LIGHT) were discovered to be involved in SL 387 hydrolysis and SL-induced seed germination; these hydrolases are D14 paralogs that act as SL 388 receptors (Conn et al. 2015b; Toh et al. 2015; Yao et al. 2017). Among them, ShHTL7 serves as 389 the most active SL receptor in Striga (Conn et al. 2015b; Yao et al. 2017). During CLIM formation, 
ShHTL7 undergoes a conformational change (like AtD14) to transduce signaling through its

391 interaction with MAX2/ShMAX2 (Yao et al. 2017).

393 Strigolactone-phytohormone crosstalk: Dynamic interplay for effective plant physiology

394 Different hormonal signaling pathways interact with one another, affecting their respective 395 signaling components (Huot et al. 2014). These dynamic interactions regulate hormonal 396 biosynthesis, response and transport, thereby helping plants control their morphology and adapt to 397 changing environmental conditions (Cheng et al. 2013). These challenging conditions include 398 severe nutritional deficiency, abiotic stress factors (i.e. salinity, heat, cold, drought and light 399 stress), and harmful biotic invasions (i.e. pathogens and pests). Phytohormone crosstalk facilitates 400 appropriate and tunable plant responses to these conditions by controlling nutrient distribution and 401 by modulating growth, developmental and defence processes. Plant stress responses are primarily 402 regulated by jasmonic acid (JA), ABA and salicylic acid (SA), whereas plant 403 growth/developmental processes are mainly governed by auxins, gibberellins and cytokinins (Huot 404 et al. 2014). SLs interact with other hormones in order to exert their impact (Saeed et al. 2017; 405 Torres-Vera et al. 2014).

Strigolactones and auxins

407 SLs inhibit shoot branching by regulating auxin transport. Compared to wild type plants, $A$. 408 thaliana max mutants show increased auxin transport due to increased PIN1/3/4/6 gene 409 transcription (Bennett et al. 2006; Lin et al. 2009). Treating Arabidopsis max mutants and rice $410 d$ warf mutants with an auxin transport inhibitor, N-1-naphthylphtalamic acid, causes inhibition of 411 bud outgrowth (Cheng et al. 2013; Lin et al. 2009). Crawford et al. (2010) reported that treatment 
412 with basal GR24 levels reduces auxin transport basipetally, as well as PIN1 accumulation in xylem

413 parenchyma cell membranes. These observations persist in biosynthetic maxl mutants but not

414 signaling $\max 2$ mutants, indicating that SLs slow down polar auxin transport stream in a MAX2-

415 dependent manner (Crawford et al. 2010).

416 Studies of auxin and max mutants showed that SLs directly affect secondary growth

417 activity, independent of auxin stacking (Agusti et al. 2011), by affecting interfascicular cambium

418 activity (Ruyter-Spira et al. 2011). Based on a quantitative study, max mutants have a $30 \%$

419 decrease in interfascicular cambium-derived tissues, concomitant with lower expression levels of

420 cambium- and cell cycle-related genes (Agusti et al. 2011). SLs regulate auxin content in the

421 primary root tip, because the primary root lengths of SL biosynthetic and signaling mutants are

422 shorter compared to wild type plants (Ruyter-Spira et al. 2011). GR24 application rescues this

423 short root phenotype in SL-deficient mutants, but not in SL-insensitive max2 mutants (Ruyter-

424 Spira et al. 2011). SLs inhibit auxin efflux by controlling PIN activity, leading to auxin

425 accumulation inside the primary root meristem cells and ultimately resulting in increased primary

426 root length (Ruyter-Spira et al. 2011). SL-auxin interaction controls root development by adjusting

427 or regulating intercellular auxin flow, auxin sensitivity and shoot-to-root transport (Mayzlish-Gati

428 et al. 2012; Omoarelojie et al. 2019). SLs also control lateral root formation by adjusting the

429 essential auxin gradient (Omoarelojie et al. 2019). Furthermore, SL-auxin interaction regulates

430 root hair elongation, whereby SLs increase intracellular auxin concentration by hindering auxin

431 efflux (Kotlai et al. 2010). Ligerot et al. (2017) suggested that a feedback loop exists in the auxin-

432 SL crosstalk. Auxins upregulate SL biosynthesis in an RMS2- (encodes PsAFB4/5 auxin receptor)

433 dependent manner, while SLs downregulate auxin levels in an RMS3- and RMS4-dependent

434 manner by downregulating auxin biosynthetic gene expression (Ligerot et al. 2017). 
436 promotes root hair elongation (Omoarelojie et al. 2019; Datta et al. 2015). In contrast to auxins,

437 SLs inhibit adventitious root (AR) formation in Arabidopsis and pea (Datta et al. 2015). AR

438 inhibition was even evident with high auxin concentration, suggesting that suppression of AR

439 formation is not due to low auxin levels (Rasmussen et al. 2012). Auxins and SLs also play a

440 crucial role during mycorrhization; auxins are associated with arbuscule formation, whereas SLs

441 are associated with presymbiotic fungal growth (Guillotin et al. 2017). The authors further found

442 that auxin content increases in roots colonized by AM fungi, and exogenous auxin application

443 promotes the colonization process. An auxin-related gene Sl-IAA27 positively controls

444 mycorrhization by regulating SL biosynthesis via NSPI (transcription factor of the D27 and MAX1

445 genes) (Guillotin et al. 2017).

\section{Strigolactones and cytokinins}

447 Cytokinins are adenine-derived plant hormones that stimulate cytokinesis and influence various

448 processes, like enhancing shoot growth, limiting root growth, and influencing axillary shoot

449 branching (Aloni et al. 2006; Werner et al. 2001). In P. sativum and A. thaliana, branching mutants

450 with increased SLs have reduced cytokinin concentrations in the xylem sap (Morris et al. 2001;

451 Foo et al. 2007). Decreased cytokinin sensitivity has also been reported in the buds of SL-

452 insensitive plants (El-Showk et al. 2013). Dun et al. (2012) reported that the SL-insensitive and

453 SL-deficient $P$. sativum rms mutants ( $r m s 4$ and $r m s 1$ ) have increased expression of the cytokinin

454 biosynthetic gene PSIPT1 in shoot nodes and internodes. Interestingly, the rms 1 mutant was more

455 sensitive to low cytokinin levels compared to wild type, when applied to the buds or supplied

456 through the vasculature (Dun et al. 2012). The authors further found that bud outgrowth is higher

457 in rms 1 mutants than wild type plants after applying low cytokinin levels, suggesting that SLs and 
cytokinins play antagonistic roles. Exogenous GR24/ cytokinin application weakened the effect of

459 cytokinins in rms 1 mutants but not in rms4 mutants, implying that SL-cytokinin interaction

460 converges at RAMOSUS4 (RMS4) (Dun et al. 2012). The cytokinin-SL antagonism is due to

461 PsBRC1, a common target of both hormones (El-Showk et al. 2013); its gene expression

462 negatively correlates with bud growth (Dun et al. 2012). Additionally, PsBRC1 gene expression is

463 enhanced by GR24 but reduced by cytokinins - a trend that persists even with cycloheximide

464 (ribosomal translation inhibitor) treatment, suggesting that new protein synthesis is not required

465 for this regulation (Dun et al. 2012). Both SLs and cytokinins act as negative regulators of lateral

466 root development; the cytokinin receptors ARR1, ARR12 and AHK3 are associated with GR24-

467 induced reduction of lateral development (Ruyter-Spira et al. 2011; Jiang et al. 2015). Genetic

468 studies show that GR24-regulated lateral development is influenced by PIN1- and PIN7-mediated

469 auxin polar transport; cytokinin treatment downregulates PIN1/PIN3/PIN5 but upregulates PIN7

470 expression (Jiang et al. 2015). Moreover, the A. thaliana max2 mutants show low cytokinin

471 catabolic gene expression $(C K X 1,2,3,5)$, reflecting the negative relationship between cytokinins

472 and SLs (Banerjee et al. 2018). In O. sativa, Duan et al. (2019) observed enhanced cytokinin levels

473 in shoot bases of $d 53$ mutants.

474 Some evidence suggests that SLs and cytokinins play important roles during drought

475 adaptation (Nishiyama et al. 2011). Analyses of cytokinin-depleted Arabidopsis mutants (CKX-

476 overexpressor), as well as signaling mutants (arrl, 10, 12), indicated that cytokinin signaling

477 negatively regulates drought acclimation (Nguyen et al. 2016). Drought tolerance mechanisms in

478 these mutants involve amplified stomatal closure, increased root-to-shoot ratio, enhanced cell

479 membrane integrity, and increased ABA hypersensitivity (Nishiyama et al. 2011). Due to the

480 undesirable role of cytokinins in drought tolerance, cytokinin biosynthesis and signaling in $A$. 
481 thaliana are suppressed during drought (Cortleven et al. 2019). Drought-induced cytokinin 482 suppression occurs through the ABA-induced transcription factor AtMYB2, and members of the 483 ABA-activated Sucrose Nonfermenting 1 (SNF1)-Related Protein Kinase 2 family (Cortleven et 484 al. 2019). In contrast to cytokinins, SLs positively regulate resilience to water stress conditions, as 485 shown in studies of Arabidopsis maxl mutants and CCD7-silenced tomato mutants (Visentin et al. 486 2016; Zhang et al. 2014). Additionally, SLs decrease stomatal density (Van Ha et al. 2014) and 487 stomatal opening during drought (Zhang et al. 2018). The max mutants also show decreased 488 response to ABA (Van Ha et al. 2014). Overall, these observations clearly indicate the contrasting 489 roles of SLs and cytokinins under drought stress conditions (Li et al. 2019).

\section{Strigolactones and gibberellins}

491 The phytohormones SLs and gibberellins (GAs) may interact during their perception and signaling, 492 acting together during plant growth and development (Marzec 2017). Remarkably, SL biosynthesis 493 can be regulated by GAs (Ito et al. 2017). GAs are involved in flowering, seed production, leaf 494 morphology and shoot/root growth (Claeys et al. 2014). Various studies have indicated that SL 495 and GA signaling are very similar. Rice semi dwarf mutants in GIBBERELLIN OXIDASE 5, 6 and

4969 exhibit an extra-branched shoot phenotype similar to SL mutants (Marzec 2017). GAs control 497 tiller number through the action of ORYZA SATIVA HOMEOBOX1 (osHB1) and TEOSINTE 498 BRANCHED1 (osTB1) transcription factors (Lo et al. 2008). SLs promote the interaction between 499 the D14 receptor and SLENDER1 (SLR1), a negative regulator of GA signaling (Nakamura et al. 500 2013). SLR1 degradation occurs in an SL-dependent manner, which parallels the GA signaling 501 pathway, where the GID1 receptor binds GA to promote interaction between GID1 and DELLA 502 proteins, eventually leading to DELLA degradation via the 26S proteasome (Marzec. 2017). 503 Additionally, gene expression databases show that $\mathrm{GA}_{3}$ treatment decreases SL biosynthetic gene 
504 expression in $O$. sativa (Ito et al. 2017). The interaction between SLs and GAs in A. thaliana is

505 inconclusive; microarray data showed varying SL biosynthetic gene expression profiles upon $\mathrm{GA}_{3}$

506 treatment (Marzec et al. 2015). In O. sativa, Zou et al. (2019) found that SL biosynthetic and

507 signaling mutants exhibit dwarfism that is rescued by GA treatment. Interestingly, these mutants

508 have less bioactive GA and decreased GA sensitivity (Zou et al. 2019). This ultimately leads to

509 reduced shoot length by downregulating genes involved in cell division and elongation (Zou et al. 510 2019).

\section{Strigolactones and abscisic acid}

512 ABA is regarded as a universal stress hormone since it regulates various abiotic stress responses.

513 Like ABA, SLs are apocarotenoid hormones so it is possible that they could also act as stress

514 hormones. Tomato ABA mutants have low SL biosynthetic gene expression, including LeCCD7

515 and $L e C C D 8$, reflecting the close harmonization between SL and ABA anabolic pathways

516 (Banerjee et al. 2018). SL-deficient Arabidopsis mutants have downregulated ABA import genes,

517 like $A B C G 22$ and $A B C G 40$, resulting in ABA hyposensitivity (Van Ha et al. 2014). It has also

518 been reported that mycorrhizal plants exposed to abiotic stresses have greater SL and ABA levels

519 (Ruiz-Lozano et al. 2016). GR24 application decreased the expression of LjNCED2 in Lotus

520 japonicus, which in turn inhibited ABA accumulation during osmotic stress (Liu et al. 2015).

521 Additionally, SL-ABA interaction is demonstrated by SLs controlling ABA-induced stomatal

522 sensitivity (Van Ha et al. 2014). SLs promote seed germination under high temperature conditions

523 by regulating both $\mathrm{ABA}$ and GAs in parasitic and non-parasitic seeds (Mostofa et al. 2018).

524 Furthermore, SL biosynthetic and signaling genes in Sesbania cannabina are upregulated by ABA

525 to cope with salt stress, while SL biosynthetic inhibitor treatment induced partial salt tolerance 
526 (Ren et al. 2018). Studies using ABA-deficient tomato mutants and CCD/NCED inhibitors suggest

527 that SL regulates ABA biosynthesis through an unknown mechanism (López-Ráez et al. 2010).

\section{Strigolactones and ethylene}

529 Certain plant growth and developmental processes involve both SL and ethylene signaling, 530 including seed germination, leaf senescence, root hair elongation and hypocotyl growth (Ueda and

531 Kusaba 2015; Cheng et al. 2013; Kapulnik et al. 2011). During light treatment, SLs upregulate

532 HY5 expression in a MAX2-dependent fashion, inhibiting hypocotyl elongation (Jia et al. 2014).

533 In contrast, ethylene promotes hypocotyl elongation by augmenting HY5 degradation via COP1

534 (Yu et al. 2013). These show the antagonistic roles of these two hormones in regulating hypocotyl

535 growth. SL-mediated root hair elongation also depends on ethylene signaling, since ethylene

536 signaling mutants (like At-etr) have reduced GR24 sensitivity (Kapulnik et al. 2011). Abolishing

537 ethylene production totally eliminates SL-mediated root hair elongation, while GR24 enhances

538 ethylene biosynthetic gene ACS2 transcription (Kapulnik et al. 2011). Moreover, SLs stimulate

539 ethylene biosynthesis in Striga seeds prior to germination (Sugimoto et al. 2003). During leaf

540 senescence, SLs activate senescence signals mediated by ethylene (Ueda and Kusaba 2015).

\section{$541 \quad$ Strigolactones and salicylic acid}

542 SA is involved in plant defence responses against various pathogens, as well as tolerance to abiotic

543 stresses (Askari and Ehsanzadeh 2015; Prodhan et al. 2018; Omoarelojie. 2019). SA-mediated

544 stress tolerance is mainly due to changes in the plant's reactive oxygen species status (Omoarelojie.

545 2019). In terms of crosstalk, SA interacts with SLs during plant-fungal symbioses (Rozpadek et

546 al. 2018). GR24 treatment results in SA build-up, whereas max2 mutants have decreased SA

547 concentrations, suggesting that SLs are involved in plant defences by inducing SA production 
548 (Rozpądek et al. 2018; Omoarelojie, 2019). In wheat, foliar application of SLs and SA

549 synergistically results in lower electrolyte leakage, higher relative leaf water content and enhanced

550 antioxidant enzyme activities during drought stress (Sedaghat et al. 2017).

\section{$551 \quad$ Strigolactones and Jasmonic acid}

552 Jasmonates are involved in secondary metabolism, wounding responses and plant-pathogen/insect

553 interactions (Yan et al. 2007; Yan and Xie. 2015). JA concentration and JA-dependent PIN11 gene

554 expression are reduced in the tomato SL biosynthetic mutant Sl-ccd8 (Torres-Vera et al. 2014).

555 Because PIN11 provides resistance in Solanum lycopersicum against Botrytis cinerea (Torres-

556 Vera et al. 2014), these observations hint at a possible interplay between these two hormones

557 during disease resistance. Although there is no direct evidence depicting SL-JA interaction, both

558 are involved together in several processes, like plant-microbe interactions, mesocotyl elongation

559 and senescence; thus, their crosstalk cannot be totally ruled out (Omoarelojie. 2019). For example,

560 Lahari et al. (2019) reported that SLs induce root-knot nematode infection in rice roots by

561 inhibiting the JA pathway. Remarkably, SL biosynthetic mutants were less prone to infection by

562 the root-knot nematode Meloidogyne graminicola (Lahari et al. 2019).

\section{Strigolactones and Karrikins}

564 Karrikins (from 'karrik' meaning smoke) or KARs are smoke-derived signals produced by burning 565 vegetation; they form through the combustion of carbohydrates (Flematti et al. 2011). Although 566 not produced in planta, they can stimulate germination of dormant seeds (De Cuyper et al. 2017)

567 - an effect attributed to the butenolide pyran moiety (Flematti et al. 2007). Unlike SLs, however, 568 KARs do not induce the germination of parasitic weeds (Conn et al. 2015b). Although they have 569 different sources and effects on plant growth and development, SLs and KARs share highly similar 
570 signaling mechanisms, which could be due to their shared butenolide structure (Morffy et al. 2016).

571 The KAI2 receptor of KARs work in the same manner as the D14 receptor of SLs (Morffy et al.

572 2016). Because KAI2 and D14 are paralogs, they share the F-box protein MAX2 during signaling

573 (De Cuyper et al. 2017). Structurally, the KAI2 receptor catalytic pocket is smaller than that of the

574 D14 receptor, which hints at the binding of smaller cognate molecules (Guo et al. 2013).

575 Phylogenetic studies have shown that KAI2 was present in basal land plants instead of D14 576 orthologs, suggesting that KAI2 is ancestral and that $D 14$ probably evolved due to KAI2 duplication 577 (Waters et al. 2012b).

578 The application of $\mathrm{KAR}_{1}, \mathrm{KAR}_{2}$ as well as rac-GR24 inhibit hypocotyl elongation in 579 Arabidopsis, with rac-GR24 having greater impact than KARs (Nelson et al. 2010; De-Cuyper et 580 al. 2017). This observation is supported by $\max 2$ mutant plants that have longer hypocotyls 581 (Stirnberg et al. 2002), a phenotype shared by mutant kai2 seedlings (Waters et al. 2012b). In 582 contrast, $\mathrm{KAR}_{1}$ and $r a c$-GR24 have antagonistic effects on cotyledon growth - karrikin promotes 583 growth while rac-GR24 negatively impacts cotyledon growth (De Cuyper et al. 2017). Mutations 584 in KAI2 and MAX2 cause skewing of A. thaliana roots, but this response is independent of SL 585 perception by the D14-MAX2 complex (Swarbreck et al. 2019). Scaffidi et al. (2014) cautioned 586 about using racemic mixtures of chemically synthesized SLs, as well as their analogs like GR24, 587 since they can activate responses that are different from natural counterparts. As reported by Liu et al. (2019), both SLs and KARs shape the morphology of the 589 exodermis. They revealed that SLs positively regulate the number of hypodermal passage cells 590 (HPC), but $d 14$ mutants surprisingly have higher HPCs (Liu et al. 2019). They further noted that, 591 in contrast to $d 14, \max 2$ mutants have decreased HPC numbers (Liu et al. 2019). In Petunia, KAI2 
592 mutation also reduces HPC numbers, indicating the critical importance of the dimeric

593 KAI2/MAX2 receptor in controlling this process (Liu et al. 2019).

\section{Strigolactones and Nitric oxide}

595 There is evidence that SLs and nitric oxide (NO) possibly interact during various stress responses 596 and developmental processes. Their interplay has mostly been studied in root systems; results 597 suggest that NO negatively and positively regulates root SL biosynthesis and signaling, 598 respectively, in a nutrient-dependent manner (Bharti and Bhatla. 2015). NO can modify proteins 599 involved in SL biosynthesis and signaling, with Arabidopsis max1-1 and max2-1 mutants having 600 increased NO levels in their root tips (Kolbert. 2019). These observations highlight the possible 601 negative impact of SLs on NO biosynthesis; however, exogenous SL application increased NO 602 production, contradicting earlier genetic studies (Kolbert. 2019). GR24 treatment results in 603 decreased NO concentration in lateral roots but increased NO concentrations in primary root tips 604 (Bharti and Bhatla. 2015). Furthermore, SLs and NO act as positive regulators of meristem activity 605 thereby enhancing root elongation (Sun et al. 2016). Endogenous NO does not influence SL 606 biosynthesis, while exogenous NO upregulates the expression of SL signaling but not biosynthetic 607 genes in O. sativa (Sun et al. 2016). In addition, exogenous SLs promote accumulation of guard 608 cell $\mathrm{H}_{2} \mathrm{O}_{2}$ and NO, leading to SLOW ANION CHANNEL-ASSOCIATED 1-mediated stomatal 609 closure (Lv et al. 2017).

610

\section{Conclusion and future prospects}

612 SLs regulate plant growth, development and stress tolerance via close crosstalk with other 613 hormones. Mechanistically, SLs elicit their response by regulating hormone content, transport and 
614 delivery between diverse plant organs and within plant tissues, and also by interacting with other

615 hormone signaling cascades. Plant responses are governed by synergistic as well as antagonistic

616 interactions of SLs with other phytohormones. Based on various physiological and molecular

617 studies, SLs are essential for plant responses to stressful environmental conditions. Due to their

618 utmost importance, continued research is needed to more lucidly understand the SL biosynthetic

619 pathway, SL signaling crosstalk with other hormones, and mechanisms by which SLs regulate

620 different stress responses, growth processes and developmental programs. Although we have

621 gained significant insights in understanding SL hormonal interplay at various levels of regulation,

622 critical knowledge gaps still need to be addressed at both cellular and molecular levels. Certain

623 functions of SLs have yet to be discovered, while further investigating the SL repressor D53 could

624 reveal its involvement in other processes. On a translational level, studying SL hormones could

625 help produce crop varieties with better nutrient allocation under limiting conditions. Long-term

626 research programs could focus on developing more resilient crops, through genetic manipulation

627 of SL quantity and response. Moreover, whether the SL receptor enzymatic activity is required for

628 downstream SL signaling and function still needs to be elucidated. Because protein-protein

629 interactions during SL signaling are unique, further research is required to fully understand SL

630 crosstalk with other hormone pathways. To gain better insights and solve pressing biological

631 problems, the next decade opens a lot of research opportunities in the exciting field of strigolactone

632 hormone biology.

633

634 Acknowledgements 
635 Mr. Kaiser Iqbal Wani, Ms. Andleeb Zehra and Ms. Sadaf Choudhary are thankful to the

636 University Grants Commission (Government of India) for providing their research fellowships.

637 Dr. Christian Danve M. Castroverde is supported by institutional research start-up funds from

638 Wilfrid Laurier University, the Natural Sciences and Engineering Research Council of Canada

639 and Canada Foundation for Innovation.

\section{Conflicts of interest}

641 The authors declare that the submitted work was not carried out in the presence of any personal, 642 professional or financial relationships that could potentially be constructed as a conflict of interest.

\section{References}

645 1. Abe S, Sado A, Tanaka K, Kisugi T, Asami K, Ota S, Seto Y (2014) Carlactone is converted 646 to carlactonoic acid by MAX1 in Arabidopsis and its methyl ester can directly interact with 647 AtD14 in vitro. PNAS 111:18084-18089

648 2. Agusti J, Herold S, Schwarz M, Sanchez P, Ljung K, Dun E A, Greb T (2011) Strigolactone 649 signaling is required for auxin-dependent stimulation of secondary growth in plants. PNAS $650 \quad 108: 20242-20247$

651 3. Akiyama K, Matsuzaki K I, Hayashi H (2005) Plant sesquiterpenes induce hyphal branching 652 in arbuscular mycorrhizal fungi. Nature 435:824

653 4. Al-Babili S, \& Bouwmeester H J (2015) Strigolactones, a novel carotenoid-derived plant 654 hormone. Annu Rev Plant Biol 66:161-186 
655 5. Alder A, Jamil M, Marzorati M, Bruno M, Vermathen M, Bigler P, Ghisla S, Bouwmeester

656 H, Beyer P, Al-Babili S (2012) The path from b-carotene to carlactone, a strigolactone-like

657 plant hormone. Science 335:1348-1351

658 6. Alder A, Holdermann I, Beyer P, Al-Babili S (2008) Carotenoid oxygenases involved in 659 plant branching catalyse a highly specific conserved apocarotenoid cleavage 660 reaction. Biochem J 416:289-296

661 7. Aloni R, Aloni E, Langhans M, Ullrich C I (2006) Role of cytokinin and auxin in shaping 662 root architecture: regulating vascular differentiation, lateral root initiation, root apical 663 dominance and root gravitropism. Ann Bot 97:883-893

664 8. Arite T, Iwata H, Ohshima K, Maekawa M, Nakajima M, Kojima M, Kyozuka J (2007) 665 DWARF10, an RMS1/MAX4/DAD1 ortholog, controls lateral bud outgrowth in rice. Plant 666 J 51:1019-1029

667 9. Arite, T, Umehara M, Ishikawa S, Hanada A, Maekawa M, Yamaguchi S, Kyozuka J (2009) 668 d14, a strigolactone-insensitive mutant of rice, shows an accelerated outgrowth of tillers. Plant Cell Physiol 50:1416-1424

10. Auldridge ME, McCarty DR, Klee HJ (2006) Plant carotenoid cleavage oxygenases and their apocarotenoid products. Curr Opin Plant Biol 9:315-321

672 11. Askari E, \& Ehsanzadeh P (2015) Effectiveness of exogenous salicylic acid on root and shoot growth attributes, productivity, and water use efficiency of water-deprived fennel genotypes. Hortic Environ Biotechnol 56:687-696

675 12. Awad AA, Sato D, Kusumoto D, Kamioka H, Takeuchi Y, Yoneyama K (2006) 676 Characterization of strigolactones, germination stimulants for the root parasitic plants Striga and Orobanche, produced by maize, millet and sorghum. Plant Growth Regul 48:221 
678 13. Banerjee A, Roychoudhury A (2018) Strigolactones: multi-level regulation of biosynthesis

679 and diverse responses in plant abiotic stresses. Acta Physiol Plant 40:86

680 14. Baz L, Mori N, Mi J, Jamil M, Kountche BA., Guo X, Al-Babili S (2018) 3-

681 Hydroxycarlactone, a novel product of the strigolactone biosynthesis core pathway. Mol

$682 \quad$ Plant 11:1312-1314

683 15. Bennett T, Sieberer T, Willett B, Booker J, Luschnig C, Leyser O (2006) The Arabidopsis MAX pathway controls shoot branching by regulating auxin transport. Curr Biol 16:553-563

685 16. Bennett $\mathrm{T}$, Leyser $\mathrm{O}$ (2014) Strigolactone signaling: standing on the shoulders of 686 DWARFs. Curr Opin Plant Biol 22:7-13

687 17. Bennett T, Liang Y, Seale M, Ward S, Müller D, Leyser O (2016) Strigolactone regulates 688 shoot development through a core signalling pathway. Biol Open 5:1806-1820

689 18. Besserer A, Puech-Pagès V, Kiefer P, Gomez-Roldan V, Jauneau A, Roy S, Séjalon-Delmas 690 N (2006) Strigolactones stimulate arbuscular mycorrhizal fungi by activating $691 \quad$ mitochondria. PLoS biology 4

692 19. Beveridge CA, Ross JJ, Murfet IC (1994) Branching mutant rms-2 in Pisum sativum 693 (grafting studies and endogenous indole-3-acetic acid levels). Plant Physiol, 104:953-959

694 20. Bharti N, Bhatla SC (2015) Nitric oxide mediates strigolactone signaling in auxin and 695 ethylene-sensitive lateral root formation in sunflower seedlings. Plant signal behav 10: e1054087

697 21. Bhattacharya C, Bonfante P, Deagostino A, Kapulnik Y, Larini P, Occhiato EG, Venturello 698 P (2009) A new class of conjugated strigolactone analogs with fluorescent properties: 699 synthesis and biological activity. Org Biomol Chem 7:3413-3420 
22. Booker J, Auldridge M, Wills S, McCarty D, Klee H, Leyser O (2004) MAX3/CCD7 is a carotenoid cleavage dioxygenase required for the synthesis of a novel plant signaling molecule. Curr Biol 14:1232-1238

23. Booker J, et al. (2005) MAX1 encodes a cytochrome P450 family member that acts downstream of $M A X 3 / 4$ to produce a carotenoid-derived branch-inhibiting hormone. Dev Cell 8:443-449

24. Borghi L, Liu GW, Emonet A, Kretzschmar T, Martinoia E (2016) The importance of strigolactone transport regulation for symbiotic signaling and shoot branching. Planta 243:1351-1360

25. Boyer FD, de Saint Germain A, Pouvreau JB, Clavé G, Pillot JP, Roux A, Heugebaert TS (2014) New strigolactone analogs as plant hormones with low activities in the rhizosphere. Mol Plant 7:675-690

712 26. Brewer PB, Yoneyama K, Filardo F, Meyers E, Scaffidi A, Frickey T, Kerr SC (2016)

713 LATERAL BRANCHING OXIDOREDUCTASE acts in the final stages of strigolactone 714 biosynthesis in Arabidopsis. PNAS 113: 6301-6306

715 27. Bürger M, Chory J (2020) The many models of strigolactone signaling. Trend Plant Sci 25: $716 \quad 395-405$

717 28. Butler LG (1995) Chemical communication between the parasitic weed Striga and its crop host: a new dimension in allelochemistry. Allelopathy Chapter 12: pp 158-168

719 29. Bythell-Douglas R, Rothfels CJ, Stevenson DW, Graham SW, Wong GKS, Nelson DC, 720 Bennett T (2017) The complex origins of strigolactone signalling in land plants. bioRxiv 721 102715 
722 30. Carlsson GH, Hasse D, Cardinale F, Prandi C, Andersson I (2018) The elusive ligand 723 complexes of the DWARF14 strigolactone receptor. J Exp Bot 69:2345-2354

724 31. Challis RJ, Hepworth J, Mouchel C, Waites R, Leyser O (2013) A role for more axillary 725 growth1 (MAX1) in evolutionary diversity in strigolactone signaling upstream of 726 MAX2. Plant Physiol 161:1885-1902

727 32. Cheng X, Ruyter-Spira C, Bouwmeester, H. (2013) The interaction between strigolactones 728 and other plant hormones in the regulation of plant development. Front Plant Sci 4:199

729 33. Chevalier F, Nieminen K, Sánchez-Ferrero JC, Rodríguez ML, Chagoyen M, Hardtke CS, 730 Cubas P (2014) Strigolactone promotes degradation of DWARF14, an $\alpha / \beta$ hydrolase 731 essential for strigolactone signaling in Arabidopsis. Plant Cell 26:1134-1150

732 34. Claeys H, De Bodt S, Inzé D (2014) Gibberellins and DELLAs: central nodes in growth 733 regulatory networks. Trends Plant Sci 19:231-239

734 35. Conn CE, and Nelson DC (2015) Evidence that KARRIKIN-INSENSITIVE2 (KAI2) 735 receptors may perceive an unknown signal that is not karrikin or strigolactone. Front Plant $736 \quad$ Sci 6:1219

737 36. Conn CE, Bythell-Douglas R, Neumann D, Yoshida S, Whittington B, Westwood JH, 738 Nelson DC (2015b) Convergent evolution of strigolactone perception enabled host detection 739 in parasitic plants. Science 349:540-543

740 37. Cook CE, Whichard LP, Turner B, Wall ME, Egley GH (1966) Germination of witchweed 741 (Striga lutea Lour.): isolation and properties of a potent stimulant. Science 154:1189-1190

742 38. Cortleven A, Leuendorf JE, Frank M, Pezzetta D, Bolt S, Schmülling T (2019) Cytokinin 743 action in response to abiotic and biotic stresses in plants. Plant Cell Environ 42:998-1018 
744 39. Crawford S, Shinohara N, Sieberer T, Williamson L, George G, Hepworth J, Leyser O 745 (2010) Strigolactones enhance competition between shoot branches by dampening auxin $746 \quad$ transport. Development 137:2905-2913

747 40. Cui X (2014) Strigolactones, Karrikins, and More: Newly Discovered Molecules Light Up $748 \quad$ Plant Signaling. Mol Plant 7:579-581

749 41. Datta SP, Prescott H, Dolan L, (2015) Intensity of a pulse of RSL4 transcription factor $750 \quad$ synthesis determines Arabidopsis root hair cell size. Nat Plants 1:15138

751 42. De Cuyperdu C, Struk S, Braem L, Gevaert K, De Jaeger G, Goormachtig S (2017)

752 Strigolactones, karrikins and beyond. Plant Cell \& Environ 40:1691-1703

753 43. de Saint Germain A, Clavé G, Badet-Denisot MA, Pillot JP, Cornu D, Le Caer JP, 754 Bonhomme S (2016) An histidine covalent receptor and butenolide complex mediates $755 \quad$ strigolactone perception. Nat Chem. Biol 12:787

756 44. Delaux PM, Xie X, Timme RE, Puech-Pages V, Dunand C, Lecompte E, Delwiche CF, 757 Yoneyama K, Bécard G, Séjalon-Delmas N (2012) Origin of strigolactones in the green $758 \quad$ lineage. New Phytol 195:857-871

759 45. Dharmasiri N, Dharmasiri S, Estelle M (2005) The F-box protein TIR1 is an auxin $760 \quad$ receptor. Nature 435:441-445

761 46. Domagalska MA, \& Leyser O (2011) Signal integration in the control of shoot 762 branching. Nat Rev Mol Cell Biol 12:211-221.

763 47. Duan J, Yu H, Yuan K, Liao Z, Meng X, Jing Y, Li J (2019) Strigolactone promotes 764 cytokinin degradation through transcriptional activation of CYTOKININ 765 OXIDASE/DEHYDROGENASE 9 in rice. PNAS 116:14319-14324 
766 48. Dun EA, de Saint Germain A, Rameau C, Beveridge CA (2012) Antagonistic action of 767 strigolactone and cytokinin in bud outgrowth control. Plant physiol 158:487-498

768 49. El-Showk S, Ruonala R, Helariutta Y (2013) Crossing paths: cytokinin signalling and $769 \quad$ crosstalk. Development 140:1373-1383

770 50. Felemban A, Braguy J, Zurbriggen MD, Al-Babili S (2019) Apocarotenoids Involved in $771 \quad$ Plant Development and Stress Response. Front Plant Sci 10

772 51. Flematti GR, Goddard-Borger ED, Merritt DJ, Ghisalberti EL, Dixon KW Trengove RD 773 (2007) Preparation of $2 \mathrm{H}$-furo [2, 3-c] pyran-2-one derivatives and evaluation of their 774 germination-promoting activity. J Agric Food Chem 55:2189-2194

775 52. Flematti GR, Scaffidi A, Dixon KW, Smith SM, Ghisalberti EL (2011) Production of the 776 seed germination stimulant karrikinolide from combustion of simple carbohydrates. J Agric $777 \quad$ Food Chem 59:1195-1198

778 53. Foo E, Morris SE, Parmenter K, Young N, Wang H, Jones A, Beveridge CA (2007) 779 Feedback regulation of xylem cytokinin content is conserved in pea and Arabidopsis. Plant $780 \quad$ Physiol 143:1418-1428

781 54. Foo E, Ferguson BJ, Reid JB (2014) The potential roles of strigolactones and 782 brassinosteroids in the autoregulation of nodulation pathway. Ann Bot 113:1037-1045

783 55. Goldwasser Y, Yoneyama K, Xie X, Yoneyama K (2008) Production of strigolactones by 784 Arabidopsis thaliana responsible for Orobanche aegyptiaca seed germination. Plant Growth $785 \quad$ Regul 55:21-28

786 56. Gomez-Roldan V, Fermas S, Brewer PB, Puech-Pagès V, Dun EA, Pillot JP, Bouwmeester, 787 H (2008) Strigolactone inhibition of shoot branching. Nature 455:189 
57. Guillotin B, Etemadi M, Audran C, Bouzayen M, Bécard G, Combier JP (2017) Sl-IAA27 regulates strigolactone biosynthesis and mycorrhization in tomato (var. MicroTom). New Phytol 213:1124-1132

58. Guo Y, Zheng Z, La Clair JJ, Chory J, Noel JP (2013) Smoke-derived karrikin perception by the $\alpha / \beta$-hydrolase KAI2 from Arabidopsis. PNAS 110:8284-8289

59. Hamiaux C, Drummond RS, Janssen BJ, Ledger SE, Cooney JM, Newcomb RD, Snowden $\mathrm{KC}$ (2012) DAD2 is an $\alpha / \beta$ hydrolase likely to be involved in the perception of the plant

60. Hayward A, Stirnberg P, Beveridge C, Leyser O (2009) Interactions between auxin and strigolactone in shoot branching control. Plant Physiol 151:400-412.

61. Holmquist M (2000) Alpha beta-hydrolase fold enzymes structures, functions and mechanisms. Curr Protein Pept Sci 1:209-235

800 62. Hou X, Rivers J, Leon P, McQuinn RP, Pogson BJ (2016) Synthesis and function of apocarotenoid signals in plants. Trends Plant Sci 21:792-803

802 63. Huot B, Yao J, Montgomery BL, He SY (2014) Growth-defense tradeoffs in plants: a 803 balancing act to optimize fitness. Mol Plant 7:1267-1287

804 64. Ito S, Yamagami D, Umehara M, Hanada A, Yoshida S, Sasaki Y, Shirasu K (2017) 805 Regulation of strigolactone biosynthesis by gibberellin signaling. Plant Physiol 174: 12501259

807 65. Jia KP, Luo Q, He SB, Lu XD, Yang HQ (2014) Strigolactone-regulated hypocotyl 808 elongation is dependent on cryptochrome and phytochrome signaling pathways in Arabidopsis. Mol Plant 7:528-540 
810 66. Jiang L, Liu X, Xiong G, Liu H, Chen F, Wang L, Yi W (2013) DWARF 53 acts as a

811 repressor of strigolactone signalling in rice. Nature 504:401

812 67. Jiang L, Matthys C, Marquez-Garcia B, De Cuyper C, Smet L, De Keyser A, Goormachtig

813 S (2015) Strigolactones spatially influence lateral root development through the cytokinin

$814 \quad$ signaling network. J Exp Bot 67:379-389

815 68. Johnson X, Brcich T, Dun EA, Goussot M, Haurogné K, Beveridge CA, Rameau C (2006)

816 Branching genes are conserved across species. Genes controlling a novel signal in pea are

817 coregulated by other long-distance signals. Plant physiol 142:1014-1026

818 69. Kagiyama M, Hirano Y, Mori T, Kim SY, Kyozuka J, Seto Y, Yamaguchi S, Hakoshima T

819 (2013) Structures of D14 and D14L in the strigolactone and karrikin signaling pathways.

$820 \quad$ Genes Cells 18:147-160

821 70. Kapulnik Y, Resnick N, Mayzlish-Gati E, Kaplan Y, Wininger S, Hershenhorn J, Koltai H 822 (2011) Strigolactones interact with ethylene and auxin in regulating root-hair elongation in $823 \quad$ Arabidopsis. J Exp Bot 62:2915-2924

824 71. Kapulnik Y, \& Koltai H (2014) Strigolactone involvement in root development, response to 825 abiotic stress, and interactions with the biotic soil environment. Plant physiol 166:560-569

826 72. Katsir L, Chung HS, Koo AJ, Howe GA (2008) Jasmonate signaling: a conserved 827 mechanism of hormone sensing. Curr Opin Plant Biol 11:428-435

828 73. Kim HI, Kisugi T, Khetkam P, Xie X, Yoneyama K, Uchida K, Yoneyama K (2014) 829 Avenaol, a germination stimulant for root parasitic plants from Avena $830 \quad$ strigosa. Phytochemistry 103:85-88

831 74. Kohlen W, Charnikhova T, Liu Q, Bours R, Domagalska MA, Beguerie S, Ruyter-Spira C 832 (2011) Strigolactones are transported through the xylem and play a key role in shoot 
architectural response to phosphate deficiency in nonarbuscular mycorrhizal host Arabidopsis. Plant Physiol 155:974-987

835 75. Kolbert Z (2019) Strigolactone-nitric oxide interplay in plants: The story has just 836 begun. Physiol Plant 165:487-497

837 76. Koltai H, Dor E, Hershenhorn J, Joel DM, Weininger S, Lekalla S, Barg, R. (2010) 838 Strigolactones effect on root growth and root-hair elongation may be mediated by auxinefflux carriers. J Plant Growth Regul 29:129-136

840 77. Koltai H, \& Beveridge CA (2013) Strigolactones and the coordinated development of shoot and root. In Long-Distance Systemic Signaling and Communication in Plants (pp. 189-204). Springer, Berlin, Heidelberg

843 78. Koltai H (2014) Receptors, repressors, PINs: a playground for strigolactone signaling. Trends Plant Sci 19:727-733

845 79. Lahari Z, Ullah C, Kyndt T, Gershenzon J, Gheysen G (2019) Strigolactones enhance root846 knot nematode (Meloidogyne graminicola) infection in rice by antagonizing the jasmonate pathway. New Phytol 224:454-465

80. Ligerot Y, de Saint Germain A, Waldie T, Troadec C, Citerne S, Kadakia N, Leyser O (2017) The pea branching RMS2 gene encodes the PsAFB4/5 auxin receptor and is involved in an auxin-strigolactone regulation loop. PLoS Genet 13:e1007089

81. Li W, Herrera-Estrella L, Tran LSP (2019) Do Cytokinins and Strigolactones Crosstalk during Drought Adaptation? Trends Plant Sci 24:669-672 to regulate shoot development in Arabidopsis via partially EAR motif-independent mechanisms. Plant Cell 28:1581-1601 
83. Lin H, Wang R, Qian Q, Yan M, Meng X, Fu Z, Yan C, Jiang B, Su Z, Li J, et al (2009) DWARF27, an iron-containing protein required for the biosynthesis of strigolactones,

84. Liu J, He H, Vitali M, Visentin I, Charnikhova T, Haider I, Cardinale F (2015) Osmotic

86. Lo SF, Yang SY, Chen KT, Hsing YI, Zeevaart JA, Chen LJ, Yu SM (2008) A novel class

85. Liu G, Stirnemann M, Gübeli C, Egloff S, Courty PE, Aubry S, Borghi L (2019) between strigolactones and ABA under abiotic stress. Planta 241:1435-1451 stress represses strigolactone biosynthesis in Lotus japonicus roots: exploring the interaction Strigolactones Play an Important Role in Shaping Exodermal Morphology via a KAI2Dependent Pathway. iScience 17:144-154 of gibberellin 2-oxidases control semidwarfism, tillering, and root development in rice. Plant Cell 20:2603-2618

87. López-Ráez JA, Charnikhova T, Gómez-Roldán V, Matusova R, Kohlen W, De Vos R, Bouwmeester H (2008) Tomato strigolactones are derived from carotenoids and their biosynthesis is promoted by phosphate starvation. New Phytol 178:863-874

88. López-Ráez JA, Kohlen W, Charnikhova T, Mulder P, Undas AK, Sergeant MJ, Bouwmeester, H. (2010). Does abscisic acid affect strigolactone biosynthesis? New Phytol 187:343-354

89. Lu T, Yu H, Li Q, Chai L, Jiang W (2019) Improving plant growth and alleviating 
peroxide synthesis and nitric oxide production in an abscisic acid-independent manner. New Phytol 217:290-304

91. Ma N, Hu C, Wan L, Hu Q, Xiong J, Zhang C (2017) Strigolactones improve plant growth, photosynthesis, and alleviate oxidative stress under salinity in rapeseed (Brassica napus L.) by regulating gene expression. Front Plant Sci 8:1671

884 92. Marzec M, Muszynska A (2015) In silico analysis of the genes encoding proteins that are involved in the biosynthesis of the RMS/MAX/D pathway revealed new roles of strigolactones in plants. Int J Mol Med 16:6757-6782

93. Marzec M (2017) Strigolactones and gibberellins: a new couple in the phytohormone world? Trends Plant Sci 22:813-815

94. Marzec M, Gruszka D, Tylec P, Szarejko I (2016) Identification and functional analysis of the $\mathrm{HvDl}$ gene involved in strigolactone signalling in Hordeum vulgare L. Physiol Plant $158: 341-355$

95. Marzec M, Brewer P (2019) Binding or Hydrolysis? How Does the Strigolactone Receptor Work? Trends Plant Sci 24:571-574

894 96. Matusova R, Rani K, Verstappen FWA, Franssen MCR, Beale MH, Bouwmeester HJ (2005) The strigolactone germination stimulants of the plant-parasitic Striga and Orobanche spp. are derived from the carotenoid pathway. Plant Physiol 139:920-934

897 97. Mayzlish-Gati E, De-Cuyper C, Goormachtig S, Beeckman T, Vuylsteke M, Brewer PB, Wininger S (2012) Strigolactones are involved in root response to low phosphate conditions in Arabidopsis. Plant Physiol 160:1329-1341

900

98. Moise AR, Al-Babili S, Wurtzel ET (2014) Mechanistic aspects of carotenoid biosynthesis. 
902 99. Morffy N, Faure L, Nelson DC (2016) Smoke and hormone mirrors: action and evolution of 903 karrikin and strigolactone signaling. Trends Genet 32:176-188

904 100. Morris SE, Turnbull CG, Murfet IC, Beveridge CA (2001) Mutational analysis of branching 905 in pea. Evidence That Rms1 and Rms5 regulate the same novel signal. Plant $906 \quad$ Physiol 126:1205-1213

907 101. Mostofa MG, Li W, Nguyen KH, Fujita M, Tran LP (2018) Strigolactones in plant adaptation 908 to abiotic stresses: An emerging avenue of plant research. Plant cell environ 41:2227-2243

909 102. Nakamura H, Xue YL, Miyakawa T, Hou F, Qin HM, Fukui K, Miyauchi Y (2013) 910 Molecular mechanism of strigolactone perception by DWARF14. Nature commun 4:1-10

911 103. Napoli CA, Ruehle J (1996) New mutations affecting meristem growth and potential in $912 \quad$ Petunia hybrida Vilm. J Hered 87:371-377

913 104. Nardini M, Dijkstra BW (1999) $\alpha / \beta$ Hydrolase fold enzymes: the family keeps growing. Curr $914 \quad$ Opin Struct Biol 9:732-737

915 105. Nelson DC, Flematti GR, Riseborough JA, Ghisalberti EL, Dixon KW, Smith SM (2010) 916 Karrikins enhance light responses during germination and seedling development in 917 Arabidopsis thaliana. PNAS 107:7095-7100

918 106. Nelson DC, Scaffidi A, Dun EA, Waters MT, Flematti GR, Dixon KW, Smith SM (2011) F919 box protein MAX2 has dual roles in karrikin and strigolactone signaling in Arabidopsis 920 thaliana. PNAS 108:8897-8902

921 107. Nguyen KH, Van Ha C, Nishiyama R, Watanabe Y, Leyva-González MA, Fujita Y, Schaller 922 GE (2016) Arabidopsis type B cytokinin response regulators ARR1, ARR10, and ARR12 923 negatively regulate plant responses to drought. PNAS 113:3090-3095 
924 108. Nishiyama R, Watanabe Y, Fujita Y, Le DT, Kojima M, Werner T, Sakakibara H (2011)

925 Analysis of cytokinin mutants and regulation of cytokinin metabolic genes reveals important

926 regulatory roles of cytokinins in drought, salt and abscisic acid responses, and abscisic acid

927 biosynthesis. Plant Cell 23:2169-2183

928 109. Omoarelojie LO, Kulkarni MG, Finnie JF, Van Staden J (2019) Strigolactones and their 929 crosstalk with other phytohormones. Annal Bot 124:749-767

930 110. Ongaro V, Leyser O (2008) Hormonal control of shoot branching. J Exp Bot 59:67-74

931 111. Prodhan MY, Munemasa S, Nahar MNEN, Nakamura Y, Murata Y (2018) Guard cell 932 salicylic acid signaling is integrated into abscisic acid signaling via the Ca2+/CPK933 dependent pathway. Plant Physiol 178:441-450

934 112. Rani K, Zwanenburg B, Sugimoto Y, Yoneyama K, Bouwmeester HJ (2008) Biosynthetic 935 considerations could assist the structure elucidation of host plant produced rhizosphere 936 signalling compounds (strigolactones) for arbuscular mycorrhizal fungi and parasitic 937 plants. Plant Physiol Biochem 46:617-626

938 113. Rasmussen A, Mason MG, De Cuyper C, et al. 2012. Strigolactones suppress adventitious 939 rooting in Arabidopsis and pea. Plant Physiol 158:1976-1987

940 114. Ren CG, Kong CC, Xie ZH (2018) Role of abscisic acid in strigolactone-induced salt stress 941 tolerance in arbuscular mycorrhizal Sesbania cannabina seedlings. BMC Plant Biol 18: 74

942 115. Rozpądek P, Domka AM, Nosek M, et al. 2018. The role of strigolactone in the cross-talk 943 between Arabidopsis thaliana and the endophytic fungus Mucor sp. Front Microbiol 9:441

944 116. Ruiz-Lozano JM, Aroca R, Zamarreño ÁM, Molina S, Andreo-Jiménez B, Porcel R, López945 Ráez JA (2016) Arbuscular mycorrhizal symbiosis induces strigolactone biosynthesis under 
drought and improves drought tolerance in lettuce and tomato. Plant Cell Environ 39: 441452

948 117. Ruyter-Spira C, Kohlen W, Charnikhova T, van Zeijl A, van Bezouwen L, de Ruijter N, 949 Verstappen F (2011) Physiological effects of the synthetic strigolactone analog GR24 on 950 root system architecture in Arabidopsis: another below ground role for strigolactones? Plant $951 \quad$ physiol 155:721-734

952 118. Ruyter-Spira C, Al-Babili S, van der Krol S, Bouwmeester H (2013) The biology of 953 strigolactones. Trends Plant Sci 18:72-83

954 119. Saeed W, Naseem S, Ali Z (2017) Strigolactones biosynthesis and their role in abiotic stress resilience in plants: A critical review. Front Plant Sci 8:1487

956 120. Scaffidi A, Waters MT, Sun YK, Skelton BW, Dixon KW, Ghisalberti EL, Smith SM (2014)

957 Strigolactone hormones and their stereoisomers signal through two related receptor proteins 958 to induce different physiological responses in Arabidopsis. Plant Physiol 165:1221-1232

959 121. Schwechheimer C, Willige BC (2009) Shedding light on gibberellic acid signalling. Curr $960 \quad$ Opin Plant Biol 12:57-62.

961 122. Sedaghat M, Tahmasebi-Sarvestani Z, Emam Y, Mokhtassi-Bidgoli A (2017) Physiological 962 and antioxidant responses of winter wheat cultivars to strigolactone and salicylic acid in 963 drought. Plant Physiol Biochem 119:59-69

964 123. Seto Y, Yamaguchi S (2014) Strigolactone biosynthesis and perception. Curr Opin Plant $965 \quad$ Biol 21: 1-6

966 124. Seto Y, Sado A, Asami K, Hanada A, Umehara M, Akiyama K, Yamaguchi S (2014) 967 Carlactone is an endogenous biosynthetic precursor for strigolactones. PNAS 111:1640968 1645 
969 125. Seto Y, Yasui R, Kameoka H, Tamiru M, Cao M, Terauchi R, Umehara M (2019)

970 Strigolactone perception and deactivation by a hydrolase receptor DWARF14. Nature

$971 \quad$ Commun 10:191

972 126. Shabek N, Ticchiarelli F, Mao H, Hinds TR, Leyser O, Zheng N (2018) Structural plasticity

973 of D3-D14 ubiquitin ligase in strigolactone signalling. Nature 563:652-656

974 127. Shinohara N, Taylor C, Leyser O (2013) Strigolactone can promote or inhibit shoot

975 branching by triggering rapid depletion of the auxin efflux protein PIN1 from the plasma

976 membrane. PLoS Biol 11(1)

977 128. Simon L, Levesque RC, Lalonde M (1993) Identification of endomycorrhizal fungi

978 colonizing roots by fluorescent single-strand conformation polymorphism-polymerase chain

979 reaction. Appl Environ Microbiol 59:4211-4215

980 129. Simons JL, Napoli CA, Janssen BJ, Plummer KM, Snowden KC (2007) Analysis of the

981 DECREASED APICAL DOMINANCE genes of petunia in the control of axillary

$982 \quad$ branching. Plant Physiol 143:697-706

983 130. Smith SM, Li J (2014) Signalling and responses to strigolactones and karrikins. Curr Opin

$984 \quad$ Plant Biol 21:23-29

985 131. Snowden KC, Simkin AJ, Janssen BJ, Templeton KR, Loucas HM, Simons JL, Klee HJ

986 (2005) The Decreased apical dominance1/Petunia hybrida CAROTENOID CLEAVAGE

987 DIOXYGENASE8 gene affects branch production and plays a role in leaf senescence, root

988 growth, and flower development. The Plant Cell 17:746-759

989 132. Snowden KC, Janssen BJ (2016) Structural biology: signal locked in. Nature 536: 402 
990 133. Song X, Lu Z, Yu H, Shao G, Xiong J, Meng X, Yao XF (2017) IPA1 functions as a 991 downstream transcription factor repressed by D53 in strigolactone signaling in rice. Cell $992 \quad$ Res $27: 1128$

993 134. Sorefan K, Booker J, Haurogné K, Goussot M, Bainbridge K, Foo E, Leyser O (2003) MAX4 and RMS1 are orthologous dioxygenase-like genes that regulate shoot branching in Arabidopsis and pea. Genes Dev 17:1469-1474

135. Soundappan I, Bennett T, Morffy N, Liang Y, Stanga JP, Abbas A, et al. (2015) SMAX1LIKE/D53 family members enable distinct MAX2-dependent responses to strigolactones and karrikins in Arabidopsis. Plant Cell 27:3143-3159

136. Stanga JP, Smith SM, Briggs WR, Nelson DC (2013) SUPPRESSOR OF MORE 1000 AXILLARY GROWTH2 1 controls seed germination and seedling development in Arabidopsis. Plant Physiol 163:318-330

1002 137. Stirnberg P, van De Sande K, Leyser HO (2002) MAX1 and MAX2 control shoot lateral 1003 branching in Arabidopsis. Development 129:1131-1141

1004 138. Sugimoto Y, Ali AM, Yabuta S, Kinoshita H, Inanaga S, Itai A (2003) Germination strategy 1005 of Striga hermonthica involves regulation of ethylene biosynthesis. Physiol Plant 119:137145

1007 139. Sun H, Bi Y, Tao J, Huang S, Hou M, Xue R, Liang Z, Gu P, Yoneyama K, Xie X, Shen Q, 1008 Xu G, Zhang Y (2016) Strigolactones are required for nitric oxide to induce root elongation in response to nitrogen and phosphate deficiencies in rice. Plant Cell Environ 39:1473-1484

140. Swarbreck SM, Guerringue Y, Matthus E, Jamieson FJ, Davies JM (2019) Impairment in 1011 karrikin but not strigolactone sensing enhances root skewing in Arabidopsis thaliana. Plant 1012 J 98:607-621 
1013 141. Tan BC, Joseph LM, Deng WT, Liu L, Li QB, Cline K, McCarty DR (2003) Molecular 1014 characterization of the Arabidopsis 9-cis epoxycarotenoid dioxygenase gene family. Plant $1015 \quad$ J 35:44-56

1016 142. Toh S, Kamiya Y, Kawakami N, Nambara E, McCourt P, Tsuchiya Y (2012) 1017 Thermoinhibition uncovers a role for strigolactones in Arabidopsis seed germination. Plant $1018 \quad$ Cell Physiol, 53:107-117

1019 143. Toh S, Holbrook-Smith D, Stogios PJ, Onopriyenko O, Lumba S, Tsuchiya Y, McCourt P 1020 (2015) Structure-function analysis identifies highly sensitive strigolactone receptors in $1021 \quad$ Striga. Science 350:203-207

1022 144. Torres-Vera R, García JM, Pozo MJ, López-Ráez JA. (2014). Do strigolactones contribute 1023 to plant defence? Mol Plant Pathol 15:211-216

1024 145. Tsuchiya Y, Yoshimura M, Sato Y, Kuwata K, Toh S, Holbrook-Smith D, et al. (2015) 1025 Probing strigolactone receptors in Striga hermonthica with fluorescence. Science 349:864$1026 \quad 868$

1027 146. Ueda H, Kusaba M (2015) Strigolactone regulates leaf senescence in concert with ethylene 1028 in Arabidopsis. Plant physiol 169:138-147

1029 147. Ueguchi-Tanaka M, Ashikari M, Nakajima M, Itoh H, Katoh E, Kobayashi M, Chow TY, 1030 Hsing YI, Kitano H, Yamaguchi I, Matsuoka M (2005) GIBBERELLIN INSENSITIVE 1031 DWARF1 encodes a soluble receptor for gibberellin. Nature 437:693-698

1032 148. Umehara M, Hanada A, Yoshida S, Akiyama K, Arite T, Takeda-Kamiya N, Magome H, 1033 Kamiya Y, Shirasu K, Yoneyama K, Kyozuka J, Yamaguchi S (2008) Inhibition of shoot 1034 branching by new terpenoid plant hormones. Nature 455:195-200 
1035 149. Umehara M, Cao M, Akiyama K, Akatsu T, Seto Y, Hanada A, Yamaguchi S (2015) 1036 Structural requirements of strigolactones for shoot branching inhibition in rice and 1037 Arabidopsis. Plant Cell Physiol 56:1059-1072

1038 150. Van Ha C, Leyva-González MA, Osakabe Y, Tran UT, Nishiyama R, Watanabe Y, 1039 Yamaguchi-Shinozaki K (2014) Positive regulatory role of strigolactone in plant responses 1040 to drought and salt stress. PNAS 111:851-856

1041 151. Visentin I, Vitali M, Ferrero M, Zhang Y, Ruyter-Spira C, Novák O, Cardinale F (2016) 1042 Low levels of strigolactones in roots as a component of the systemic signal of drought stress 1043 in tomato. New Phytol 212:954-963

1044 152. Waldie $\mathrm{T}$, McCulloch H, Leyser O (2014) Strigolactones and the control of plant 1045 development: lessons from shoot branching. Plant J 79:607-622

1046 153. Wang JY, Haider I, Jamil M, Fiorilli V, Saito, Y, Mi J, Balakrishna A (2019) The 1047 apocarotenoid metabolite zaxinone regulates growth and strigolactone biosynthesis in 1048 rice. Nature commun 10:810

1049 154. Wang Y, Shang L, Yu H, Zeng L, Hu J, Ni S, Wang L (2020a) A strigolactones biosynthesis 1050 gene contributed to the Green Revolution in rice. Mol Plant

1051 155. Wang L, Xu Q, Yu H, Ma H, Li X, Yang J, Li J (2020b) Strigolactone and karrikin signaling 1052 pathways elicit ubiquitination and proteolysis of SMXL2 to regulate hypocotyl elongation 1053 in Arabidopsis thaliana. Plant Cell.

1054 156. Waters MT, Brewer PB, Bussell JD, Smith SM, Beveridge CA (2012a) The Arabidopsis 1055 ortholog of rice DWARF27 Acts upstream of MAX1 in the control of plant development by $1056 \quad$ strigolactones. Plant Physiol 159:1073-1085 
1057 157. Waters MT, Nelson DC, Scaffidi A, Flematti GR, Sun YK, Dixon KW, Smith SM (2012b)

1058 Specialisation within the DWARF14 protein family confers distinct responses to karrikins

1059 and strigolactones in Arabidopsis. Development 139:1285-1295

1060 158. Waters MT, Scaffidi A, Moulin SL, Sun YK, Flematti GR, Smith SM (2015) A Selaginella

1061 moellendorffii ortholog of KARRIKIN INSENSITIVE2 functions in Arabidopsis

1062 development but cannot mediate responses to karrikins or strigolactones. Plant Cell 27:

$1063 \quad 1925-1944$

1064 159. Waters MT, Gutjahr C, Bennett T, Nelson D (2017) Strigolactone signalling and evolution. 1065 Annu Rev Plant Biol 68

1066 160. Werner T, Motyka V, Strnad M, Schmulling T (2001) Regulation of plant growth by 1067 cytokinin. Proc Natl Acad Sci USA 98:10487-92

1068 161. Xie X, Yoneyama K, Yoneyama K (2010) The strigolactone story. Annu Review 1069 Phytopathol 48:93-117

1070 162. Yamaguchi S (2008) Gibberellin metabolism and its regulation. Annu Rev Plant Biol 59: $1071 \quad 225-251$

1072 163. Yan Y, Stolz S, Chételat A, Reymond P, Pagni M, Dubugnon L, Farmer EE (2007) A 1073 downstream mediator in the growth repression limb of the jasmonate pathway. The Plant $1074 \quad$ Cell 19:2470-2483

1075 164. Yan C, Xie D (2015) Jasmonate in plant defence: sentinel or double agent? Plant Biotechnol $1076 \quad$ J $13: 1233-1240$

1077 165. Yao R, Ming Z, Yan L, Li S, Wang F, Ma S, Yu C, Yang M, Chen L, Chen L, et al. (2016) 1078 DWARF14 is a non-canonical hormone receptor for strigolactone. Nature 536:469-473 
166. Yao R, Wang F, Ming Z, Du X, Chen L, Wang Y, Xie D (2017) ShHTL7 is a non-canonical receptor for strigolactones in root parasitic weeds. Cell Res 27:838

1081 167. Yu Y, Wang J, Zhang Z, et al. (2013) Ethylene promotes hypocotyl growth and HY5 1082

1083 degradation by enhancing the movement of COP1 to the nucleus in the light. PLoS Genet

168. Yoneyama K, Xie X, Sekimoto H, Takeuchi Y, Ogasawara S et al. (2008) Strigolactones, 1085

169. Yoneyama K, Xie X, Yoneyama K, Kisugi T, Nomura T, Nakatani Y, McErlean CS (2018) Which are the major players, canonical or non-canonical strigolactones?. J Exp Bot 69:2231-

170. Yoneyama K, Akiyama K, Brewer PB, Mori N, Kawano-Kawada M, Haruta S, Beveridge CA (2020) Hydroxyl carlactone derivatives are predominant strigolactones in Arabidopsis. Plant Direct 4:e0219

171. Zhang Y, Van Dijk AD, Scaffidi A, Flematti GR, Hofmann M, Charnikhova T, Smith SM (2014) Rice cytochrome P450 MAX1 homologs catalyze distinct steps in strigolactone biosynthesis. Nat Chem Biol 10:1028

172. Zhang Y, Lv S, Wang G (2018) Strigolactones are common regulators in induction of stomatal closure in planta. Plant Signal Behav 13, e1444322.

1098 173. Zhao LH, Zhou XE, Wu ZS, Yi W, Xu Y, Li S, Xu TH, Liu Y, Chen RZ, Kovach A, Kang 1099 Y, Hou L, He Y, Xie C, Song W, Zhong D, Xu Y, Wang Y, Li J, Zhang C, Melcher K, Xu 1100 HE (2013) Crystal structures of two phytohormone signal-transducing $\alpha / \beta$ hydrolases: 1101 karrikin-signaling KAI2 and strigolactone-signaling DWARF14. Cell Res 23: 436-439 
1102 174. Zhao J, Wang T, Wang M, Liu Y, Yuan S, Gao Y, Wan J (2014) DWARF3 participates in 1103 an SCF complex and associates with DWARF14 to suppress rice shoot branching. Plant Cell $1104 \quad$ Physiol 55:1096-1109

1105 175. Zhao LH, Zhou XE, Yi W, Wu Z, Liu Y, Kang Y, Scaffidi A (2015) Destabilization of 1106 strigolactone receptor DWARF14 by binding of ligand and E3-ligase signaling effector 1107 DWARF3. Cell Res 25:1219

1108 176. Zheng Z., de Saint Germain A., Chory J. (2014) Unfolding the mysteries of strigolactone 1109 signaling. Mol Plant 7:934

1110 177. Zheng K, Wang X, Weighill DA, Guo HB, Xie M, Yang Y, Muchero W (2016)

1111 Characterization of DWARF14 genes in Populus. Sci Rep 6:21593

1112 178. Zhou F, Lin Q, Zhu L, Ren Y, Zhou K, Shabek N, Ma W (2013) D14-SCF D3-dependent 1113 degradation of D53 regulates strigolactone signalling. Nature 504:406-410

1114 179. Zou X, Wang Q, Chen P, Yin C, Lin Y (2019) Strigolactones regulate shoot elongation by 1115 mediating gibberellin metabolism and signaling in rice (Oryza sativa L.). J Plant $1116 \quad$ Physiol 237:72-79

1117 180. Zwanenburg B, Mwakaboko AS, Reizelman A, Anilkumar G, Sethumadhavan D (2009) 1118 Structure and function of natural and synthetic signalling molecules in parasitic weed 1119 germination. Pest Manag Sci 65:478-491 


\section{Figure legends:}

1126 Fig. 1. Diverse roles of SLs in overall plant growth, development and resilience.

1127 Fig. 2. The SL biosynthetic pathway showing key enzymes and intermediates.

1128 Fig. 3. The SL signaling mechanism showing receptor complex formation and protein 1129 modifications.

1130

1131 
1132

1133

1134

1135

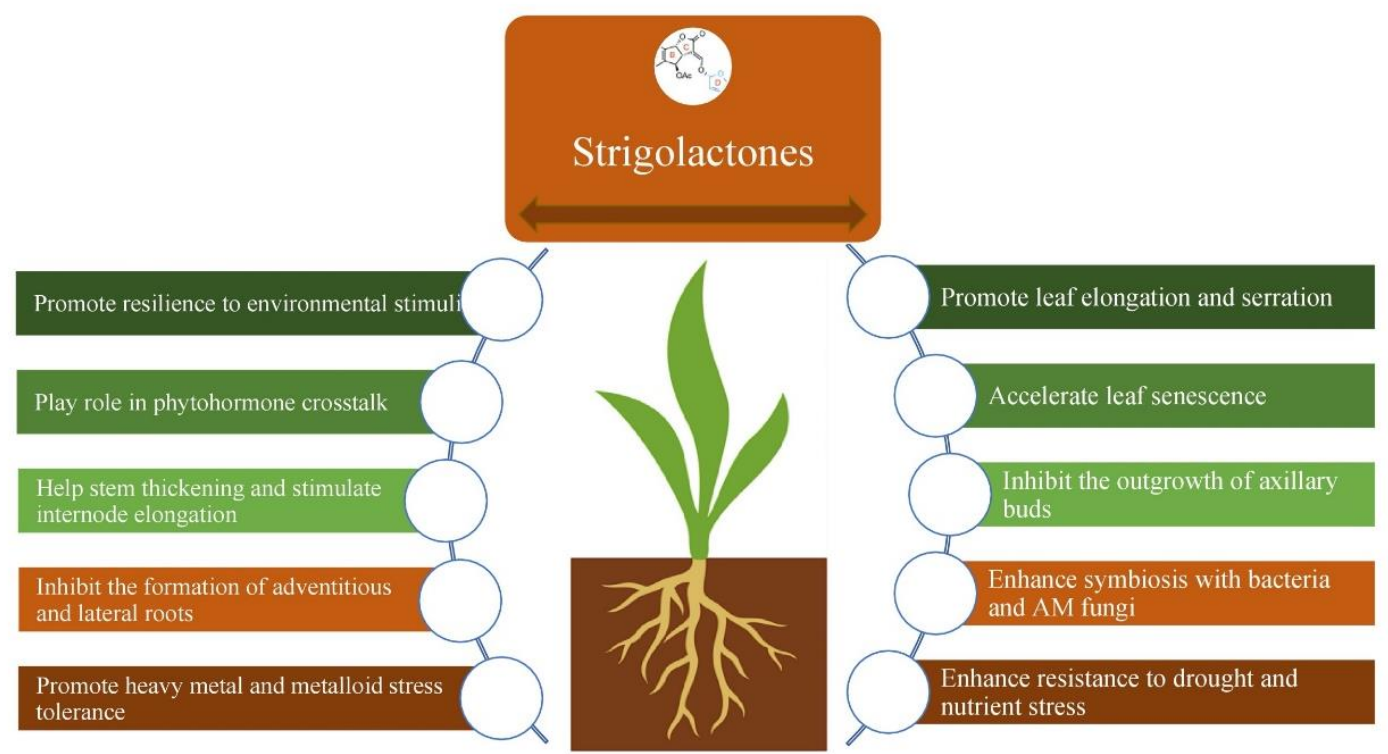

1136

1137

1138

1139

1140

1141

1142

1143

1144

1145

1146

1147

1148

1149

1150

1151

1152

1153

1154

1155

1156

1157

1158

1159

1160

1161

1162 


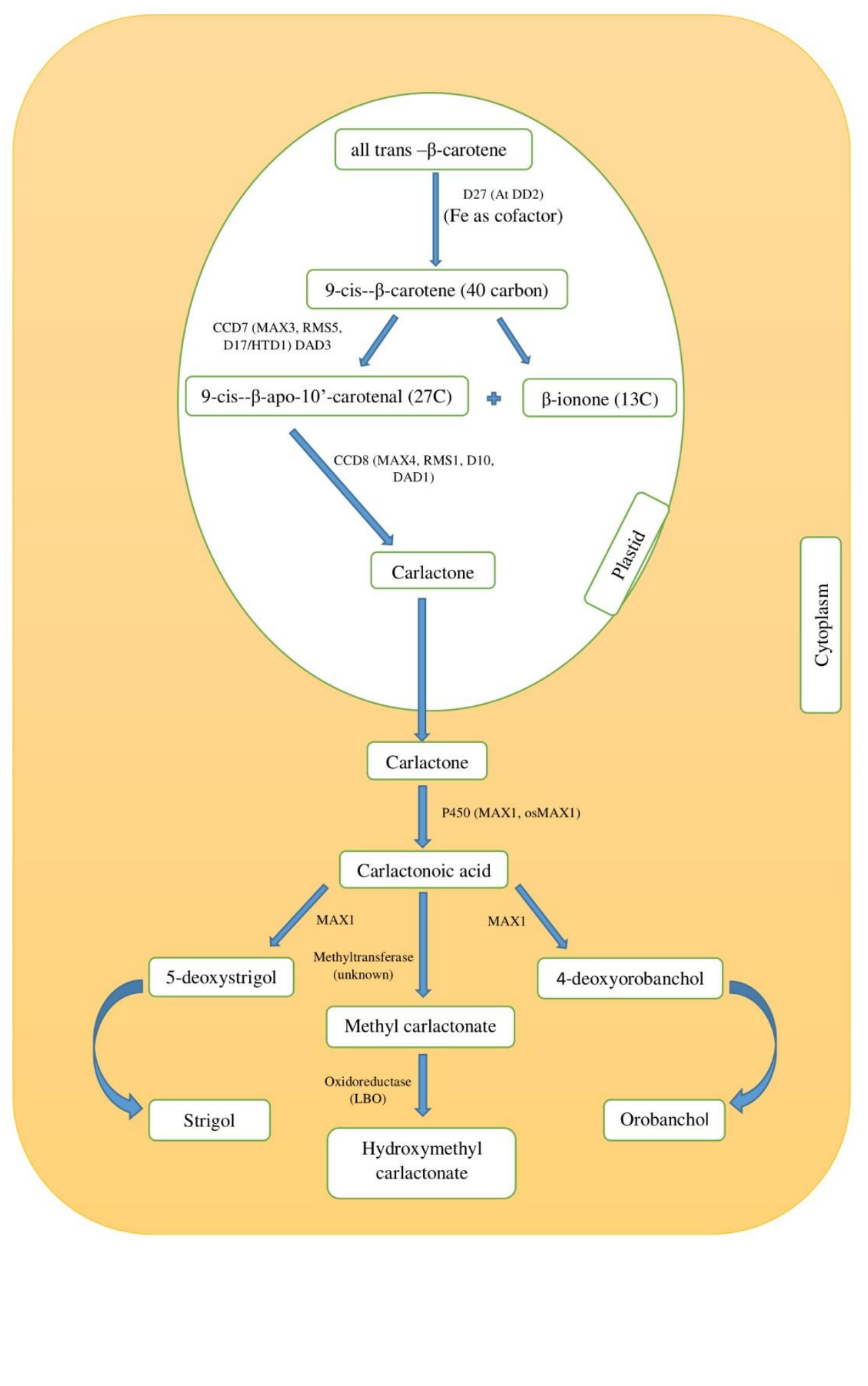


1171

1172

1173

1174

1175

1176

1177

1178

Fig. 3
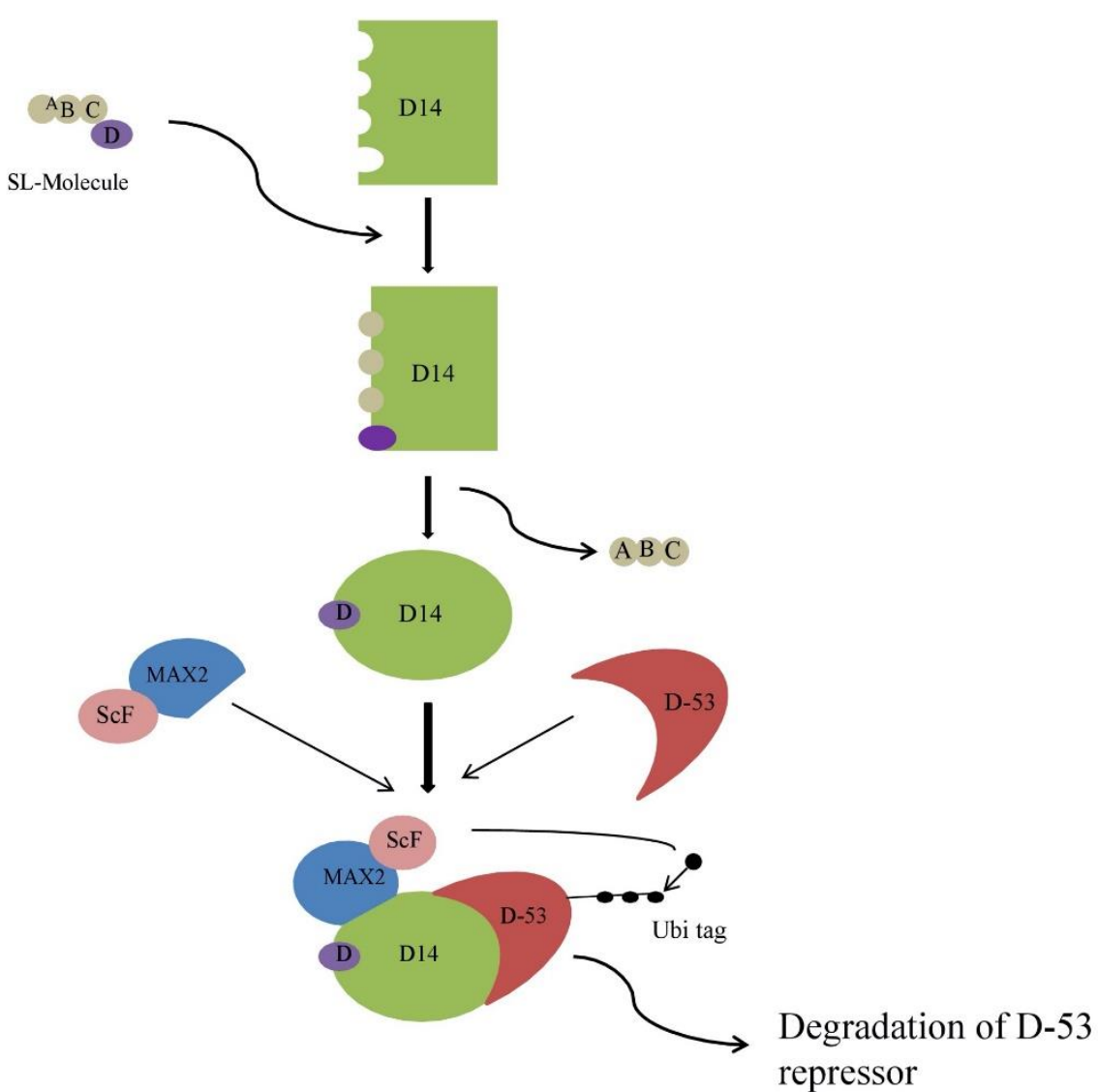